\title{
High-Power Laser Systems of UV and Visible Spectral Ranges
}

\author{
Nikolay Ivanov, Valery Losev, Yury Panchenko and \\ Viktor Tarasenko
}

Additional information is available at the end of the chapter

http://dx.doi.org/10.5772/intechopen.71455

\begin{abstract}
Three high-power excimer laser systems with apertures of 25 and $40 \mathrm{~cm}$ of the output laser beam are described. The first and second laser systems consist of four and five excimer lasers, respectively. Third system consists of Ti:Sa front end and $\mathrm{XeF}(\mathrm{C}-\mathrm{A})$ amplifier. The experimental results of the generation of the high-quality and high-power laser pulses are presented. Laser beams with pulse energy of up to $330 \mathrm{~J}(308 \mathrm{~nm}, 250 \mathrm{~ns})$ and peak power $14 \mathrm{TW}(450 \mathrm{~nm}, 50 \mathrm{fs})$ were obtained.
\end{abstract}

Keywords: excimer laser systems, discharge, e-beam, hybrid laser system, laser pulse

\section{Introduction}

Laser systems on the noble-gas halides are the most powerful and effective sources of the coherent radiation in the UV spectral range. Nowadays, these systems serve as a unique means for solving fundamental and applied problems (e.g., inertial nuclear fusion, the physics of the interaction of the superintense radiation with matter, the generation of the $x$-ray radiation, the acceleration of particles in the presence of superstrong electromagnetic field, etc.).

Nike (United States) is the most powerful excimer laser system, generating radiation pulses with energy of up to $5 \mathrm{~kJ}$ at a pulse duration of $240 \mathrm{~ns}$ on full width half maximum (FWHM) and a wavelength of $248 \mathrm{~nm}$ [1]. The aperture of the output amplifier of this system is $60 \times 60 \mathrm{~cm}$. The system was created and applied to solve the problem of laser thermonuclear fusion. It is used in the experiments on the generation of high-power nanosecond pulses and their interaction with a target. The second largest excimer laser system (Super-Ashura) was created in Japan [2]. The aperture size of the output amplifier is $61 \mathrm{~cm}$. This system generates radiation pulses with an energy 
of up to $3.7 \mathrm{~kJ}$ ( $\mathrm{KrF}$ molecule) at a pulse duration of about $240 \mathrm{~ns}$. The Super-Ashura system is used for experiments on the generation of high-power nanosecond and picosecond pulses and the interaction of these pulses with matter. The Titania laser system, created in England, employs $\mathrm{KrF}$ molecules and generates pulses with an energy of about $1 \mathrm{~kJ}$ and a pulse duration of about $150 \mathrm{~ns}$ [3]. The aperture size of the output amplifier is $42 \mathrm{~cm}$. This system is used for experiments on the generation of high-power picosecond and femtosecond pulses and their interaction with matter.

Russian institutions also develop and create the powerful excimer lasers and laser systems [4-12]. In particular, a KrF laser system with a Garpun output unit (Institute of Physics, Russian Academy of Sciences) has an output aperture with a size of $16 \times 18 \mathrm{~cm}$ and one generates radiation pulses with energy of $80 \mathrm{~J}$ and pulse duration of $100 \mathrm{~ns}$ [12].

The excimer lasers developed at the High-Current Electronics Institute (HCEI), Siberian Division, Russian Academy of Sciences, generate pulses with an energy of greater than $100 \mathrm{~J}$ [4-6, 8-11]. The two most powerful XeCl lasers (308 nm) with apertures of 40 [8] and $60 \mathrm{~cm}$ [9-11] generate pulses with energies of $660 \mathrm{~J}$ and $1.9 \mathrm{~kJ}$ and pulse durations of about 350 and 250 ns, respectively.

At the end of the nineteenth century, the hybrid approach to femtosecond pulse amplification was developed. To amplify femtosecond pulses, the rare-gas-halide excimer media excited by a high-voltage discharge or an electron beam were used. In these media, the laser transitions between the excited B-state and ground weakly bound or weakly repulsive X-state of ArF, $\mathrm{XeCl}, \mathrm{XeF}$, or $\mathrm{KrF}$ excimer molecules were used [13]. However, $\mathrm{B}-\mathrm{X}$ transitions have rather narrow gain bandwidths (broadest bandwidth of $\Delta \lambda \approx 2 \mathrm{~nm}$ ) and rather small saturation fluence $\left(\varepsilon_{\text {sat }} \approx 1 \mathrm{~mJ} / \mathrm{cm}^{2}\right)$. To realize high-output peak powers, (more than $1 \mathrm{TW}$ ) large apertures are required. Furthermore, a high gain of the amplifiers limits the temporal contrast of output radiation at the level of $10^{2}-10^{3}[14]$. The highest peak power of output radiation reached in traditional rare-gas-halide excimer amplifiers does not exceed $\sim 4$ TW [15].

A photodissociative-driven $\mathrm{XeF}(\mathrm{C}-\mathrm{A})$ medium has a wide amplification band $(\sim 60 \mathrm{~nm})$ in $475 \mathrm{~nm}$ range and a high saturation fluence of $\sim 0.05 \mathrm{~J} / \mathrm{cm}^{2}$ unlike the traditional excimer molecules on the B-X transition [16]. At present, the development of ultra-high-power laser systems with a pulse duration of 10-100 fs is based mainly on near-infrared solid-state Ti:sapphire or parametric amplifiers. In these systems, positively chirped pulses, i.e., stretched in time (up to $0.5-1 \mathrm{~ns}$ ) by linear frequency modulation [17], are amplified, and following its temporal recompression, the initial duration is realized. A pulse stretching allows avoiding nonlinear effects related to self-focusing. The pulses are normally stretched $\sim 10^{4}$ times, and for pulse recompression, a vacuum compressor based on diffraction gold-coated gratings is used.

An alternative approach to the design of multiterawatt and petawatt femtosecond laser systems has been developed at the Lebedev Physical Institute (Moscow, Russia) [18, 19], LP3 Laboratory of the Marseille University (Marseille, France) [20-22], and Institute of High Current Electronics (Tomsk, Russia) [23-25]. This approach is based on a solid-state femtosecond front-end and a photochemical $\mathrm{XeF}(\mathrm{C}-\mathrm{A})$ boosting amplifier with a gaseous active medium [26, 27]. The advantage of this hybrid (solid/gas) design is that due to the much lower optical nonlinearity of gas compared to solids, the admissible factor of stretching femtosecond pulse is three orders of magnitude smaller than for solid-state systems. This allows the amplification of picosecond chirped pulses and their subsequent compression by 
simpler and more efficient methods. The compression of negatively chirped pulses can be realized in bulk glass with positive group velocity dispersion. In addition, the hybrid laser system operates in the visible spectral range, which may be advantageous in some applications to strong-field laser-matter interaction [28]. The gaseous nature of the active medium also allows easy scaling of hybrid laser systems. At the Institute of High Current Electronics (HCEI) SB RAS, the multiterawatt hybrid laser system THL-100 based on a photochemicaldriven $\mathrm{XeF}(\mathrm{C}-\mathrm{A})$ boosting amplifier with a $24 \mathrm{~cm}$ aperture was developed.

In this work, we describe three high-power excimer laser systems developed at HCEI and based on wide-aperture lasers with the output laser beam sizes $25 \times 25 \mathrm{~cm}$ [6], $40 \mathrm{~cm}$ (diameter) [8], and $25 \mathrm{~cm}[29,30]$, respectively. The experimental results obtained on these systems are presented.

\section{MELS-4 k laser system}

\subsection{Experimental method and equipment}

The MELS-4 $\mathrm{k}$ laser system consists of the master complex, preamplifier, and a UFL-100 M amplifier [7]. The master complex contains two electric-discharge $\mathrm{XeCl}$ lasers. One of them serves as the master oscillator. The combination of the two lasers makes it possible to vary the parameters of the output radiation. In particular, for single-pass lasing, the radiation energy is $E=15 \mathrm{~mJ}$, the spectral line half width is $\Delta v=0.01 \mathrm{~cm}^{-1}$, and the pulse FWHM is $50 \mathrm{~ns}$. In the case of injection locking, the parameters of the output beam are $\mathrm{E}=100 \mathrm{~mJ}, \Delta v=0.01 \mathrm{~cm}^{-1}$, and $\tau=100 \mathrm{~ns}$, while more than $50 \%$ of the radiation energy is concentrated inside the diffraction angle [31]. In the case of double-pass lasing with the phase conjugation, the parameters are $E=50 \mathrm{~mJ}, \Delta v=0.01-0.4 \mathrm{~cm}^{-1}$, and $\tau=30 \mathrm{~ns}$, and the divergence is close to the diffractionlimited $Q_{d}$ [32]. When the pulse is compressed to a pulse duration of 1-2 ns upon stimulated Brillouin scattering, the parameters are $E=10 \mathrm{~mJ}, \Delta v=0.01 \mathrm{~cm}^{-1}$, and $Q_{d}$ [33].

The preamplifier represents an electric-discharge lase with an active volume of $6 \times 11 \times 80 \mathrm{~cm}^{3}$ (Figure 1). This laser consists of a metal housing that contains the dielectric laser chamber, capacitors with a total capacitance of $368 \mathrm{nF}$ that are directly connected to the electrodes, and the x-ray source. A discharge gap and a storage capacitor $(0.4 \mu \mathrm{F})$ are placed outside. The laser mixture $\mathrm{Ne} / \mathrm{Xe} / \mathrm{HCl}=1000 / 10 / 1$ is photo-ionized at a pressure of $2-4 \mathrm{~atm}$. The storage capacitor is connected to the discharge gap, and $300 \mathrm{~ns}$ prior to the moment when the voltage across the electrodes reaches the maximum value, the x-ray source is switched on. The radiation of this source initiates the discharge. The $\mathrm{x}$-ray radiation is injected through a stainless steel grid with a geometrical transparency of $50 \%$. The doze inside the laser chamber is about $25 \mathrm{mR}$. The laser energy amounts to $6-10 \mathrm{~J}$ at a pulse duration of $\tau=80-160 \mathrm{~ns}$.

The active volume of the main amplifier is $25 \times 25 \times 100 \mathrm{~cm}^{3}$. The gas is excited by two electron beams [6]. The electron accelerators are placed at the top and bottom of the laser chamber, which has an internal volume of $360 \mathrm{l}$. In each accelerator, the vacuum diode and the high-voltage generator are placed in a single metal housing. The cathode of the vacuum diode is directly fixed on the last stage of the high-voltage generator. The maximum energy of the laser with a plane-parallel cavity is $210 \mathrm{~J}$, and the pulse duration is $\tau=250 \mathrm{~ns}$. In the amplification mode, the windows of the laser chamber are tilted at an angle of $10^{\circ}$ relatively to the optical axis. 


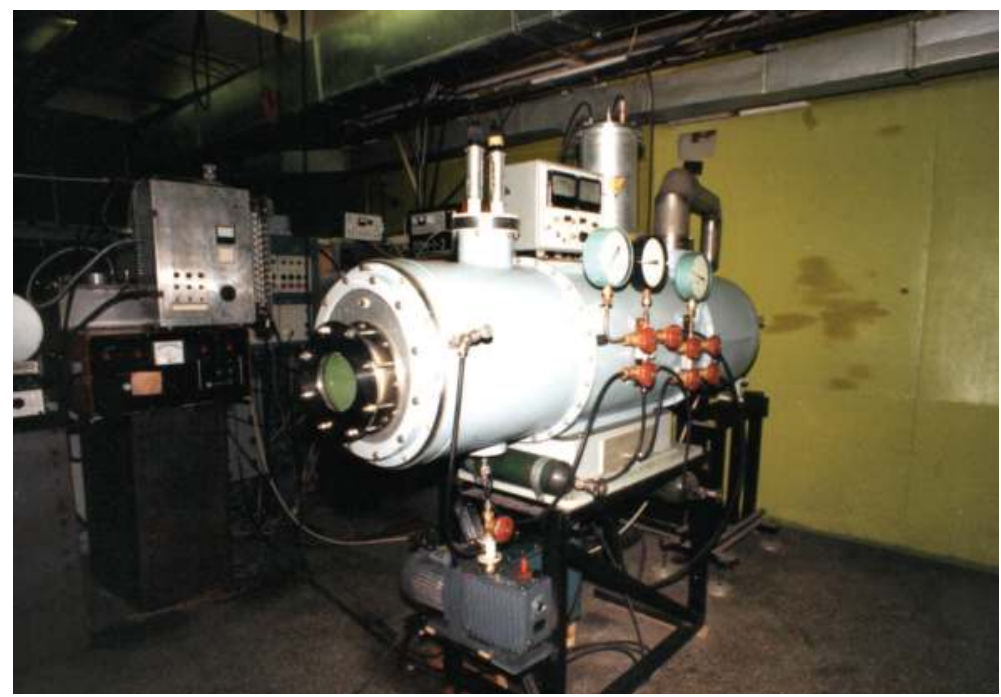

Figure 1. Photograph of the electric-discharge preamplifier.

To start the master complex and to lock it to the amplifiers, we employ a high-voltage pulsed oscillator. The switch on time of the master oscillator and the amplifier is controlled using the cable and artificial delay lines. When the radiation is amplified in the laser system, the following condition is satisfied: the maximum intensity of the input pulse coincides with the pump power maximum in each of the amplifiers.

To determine the wave-front distortions of the amplified beam on the optical elements, we calculate the optical path taking into account the positions of the elements and the surface finish. For the optical elements with a diameter of up to $100 \mathrm{~mm}$, the wave-front distortion of the laser beam is no greater than $\lambda / 4$. For a diameter $\mathrm{D}$ of greater than $100 \mathrm{~mm}$, the distortion is no greater than $\lambda$ (wave length).

To find the energy parameters of the radiation, we numerically simulate the amplification modes. For this purpose, we employ the one-dimensional model developed at HCEI based on the system of nonstationary equations for the concentrations of the excimer molecules and the photon fluxes [7].

\subsection{Calculated parameters of the amplified radiation}

The short spontaneous lifetime (10-14 ns) of the upper laser level of excimer molecules and a high small signal gain $\mathrm{g}_{0}\left(0.05-0.1 \mathrm{~cm}^{-1}\right)$ lead to a relatively high level of amplified spontaneous emission (ASE). The purpose of the calculations is to predict the output parameters of the laser system with allowance for the ASE in the optical schemes.

The output characteristics of the radiation are calculated for the preamplifier and the main amplifier where the ASE effect is maximal. All of the intensity distributions are presented for a time-constant pump power. Figure 2 demonstrates the radiation intensity distribution with respect to the length of the preamplifier active medium for various intensities of the input 
signal. The calculations are performed for the following initial parameters of the active medium: $\mathrm{g}_{0}=0.08 \mathrm{~cm}^{-1}, \alpha=0.015 \mathrm{~cm}^{-1}, \tau_{\mathrm{ef}}=2.5 \mathrm{~ns}$, and $\sigma=4 \times 10^{-16} \mathrm{~cm}^{2}$, where $\alpha$ is absorption coefficient, $\tau_{\mathrm{ef}}$ is the efficient life time of $\mathrm{XeCl}^{*}$ molecule, and $\sigma$ is the stimulated emission cross section The spontaneous lifetime of the $\mathrm{XeCl}^{*}$ molecules is $\tau_{\mathrm{s}}=14 \mathrm{~ns}$. Based on the results of the calculations and the input pulse duration, we may conclude that an input energy of about $10 \mathrm{~mJ}$ is sufficient for the saturation of the amplifier at two passes. In this case, the intensity of the amplified signal as about $2 \mathrm{I}_{\mathrm{s}}$ ( $\mathrm{I}_{\mathrm{s}}$ is the saturation intensity). For input signals with an intensity of no less than $100 \mathrm{~kW} / \mathrm{cm}^{2}$, it is expedient to employ single-pass amplification. In general, the ASE effect is weak provided that the input intensity is more than $2 \mathrm{~kW} / \mathrm{cm}^{2}$ at the given parameters and sizes of the active medium.

For the main amplifier with a lower pump power, the parameters of the active medium are as follows: $g_{0}=0.065 \mathrm{~cm}^{-1}, \alpha=0.0145 \mathrm{~cm}^{-1}, \tau_{\mathrm{ef}}=3 \mathrm{~ns}, \sigma=4 \times 10^{-16} \mathrm{~cm}^{2}$, and $\tau_{\mathrm{s}}=14 \mathrm{~ns}$ [7]. Figure $3 \mathrm{dem}$ onstrates the intensity distributions of the amplified radiation and ASE for the single-pass (a) and double-pass (b) configurations. It is seen that the ASE effect is significantly stronger than the effect in the preamplifier in spite of the comparable values of the product $\mathrm{gL}$, where $\mathrm{L}$ is active medium length and $g$ is the gain coefficient. For example, at a kilowatt level of the input signal, the ASE intensity at the output of the main preamplifier is close to the intensity of the amplified radiation, so that the latter cannot effectively make use of the population inversion. Note that the one-dimensional model slightly overestimates the signal-to-noise ratio (especially in the case of double-pass amplification). Therefore, the real ASE effect should be even stronger. Thus, the minimum intensity of the input signal of the main amplifier should be about $10 \mathrm{~kW} / \mathrm{cm}^{2}$. At a pulse duration of $80 \mathrm{~ns}$, the corresponding energy density is about $1 \mathrm{~mJ} / \mathrm{cm}^{2}$. In this case, preference should be given to the double-pass configuration with regard to the energy of the amplified radiation.

In the calculations, we employ the nonstationary model and, therefore, observe the shape of the desired signal and the ASE pulse shape [7]. The pulses at the input and output of the

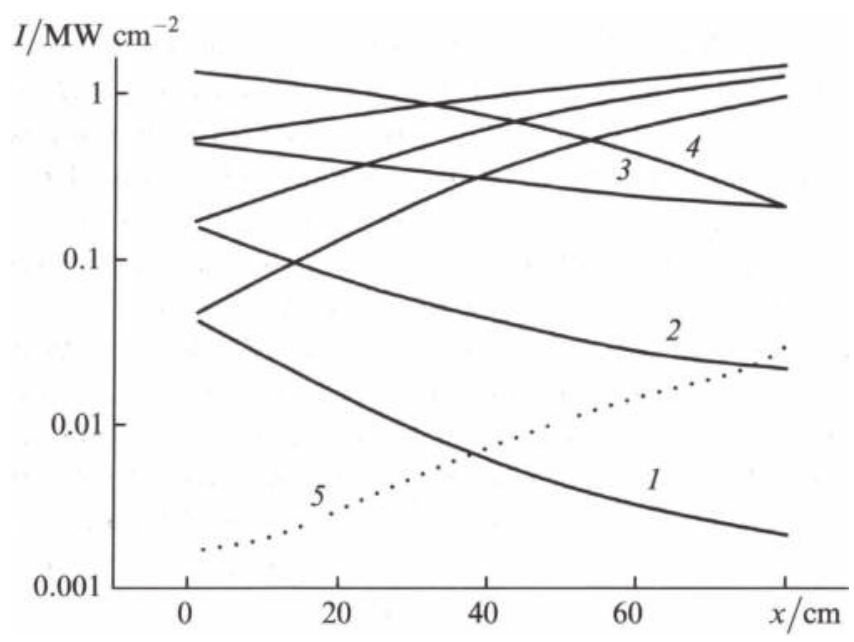

Figure 2. Intensity distributions of (1)-(4) the amplified signals and (5) the total ASE for (1)-(3) and (5) the double-pass and (4) single-pass amplification in the active medium of the preamplifier. The signal traveling from the right-hand side to the left-hand side is reflected by the mirror on the left-hand side. Curve 5 corresponding to the ASE is presented for the conditions of curve 1 . 
active medium may substantially differ from each other. This difference depends on the difference between the radiation and pump pulse shapes, on the steepness of the pulse edges and the length of the active medium.
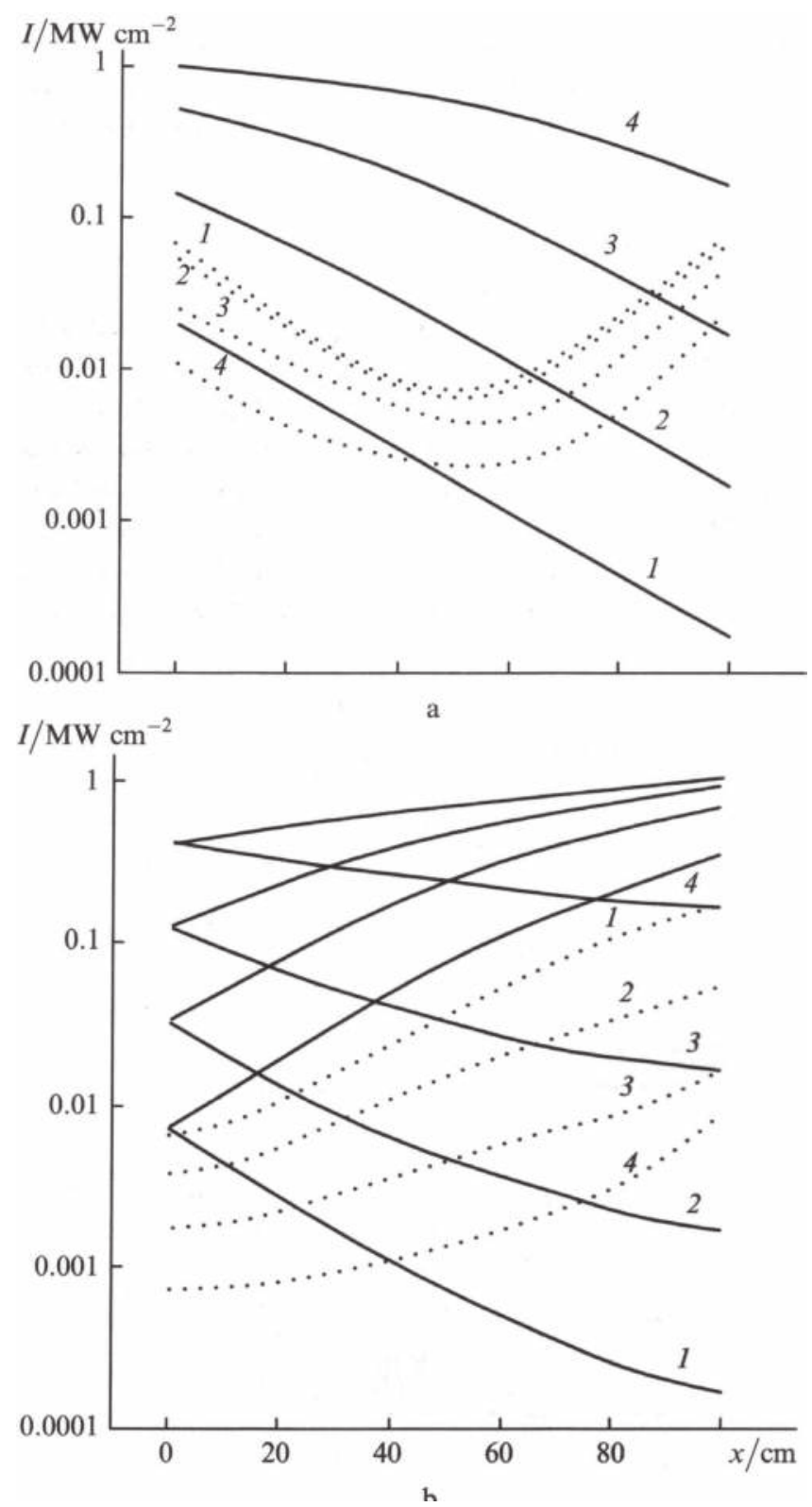

Figure 3. Intensity distributions of (solid lines 1-4) the amplified signals and (dashed lines 1-4) the total ASE for (a) one and (b) two passes in the active medium of the main amplifier. 


\subsection{Experimental results}

The experiments on the amplification of radiation in the laser systems are performed at a pulse duration of $\tau_{0.5}=80 \mathrm{~ns}$. The purpose of the experiments is to measure the energy characteristics of the amplifiers and to determine the wave-front distortions in the optical path. Figure 4 shows the experimental optical scheme. In the case under consideration, the amplified beam diverges owing to the presence of the negative lens 5. This makes it possible, on the one hand, to increase the output energy of the first stage and, on the other hand, to relatively easily match the apertures of the electric-discharge amplifiers. To match the beam size with the aperture of the output amplifier, we additionally employ telescope 6 , which also provides for the spatial filtering of the radiation.

Table 1 demonstrates the measured output radiation energies and divergences for each of the amplification stages. Here, $A$ is the beam size at the amplifier output; $E_{\text {in }}$ and $E_{\text {out }}$ are the input and output energies, respectively; and $E_{d}$ is the energy inside the angle $Q_{d}$. The larger beam size in the preamplifier and amplifier corresponds to the presence of the telescope. The difference between the output and input energies of the stages is related to the loss at the turning mirrors and the loss resulting from the aperture matching.

It is seen from Table 1 that the radiation divergence at the output of the first stage is close to the diffraction-limited divergence. Nevertheless, a worsening of the discharge homogeneity

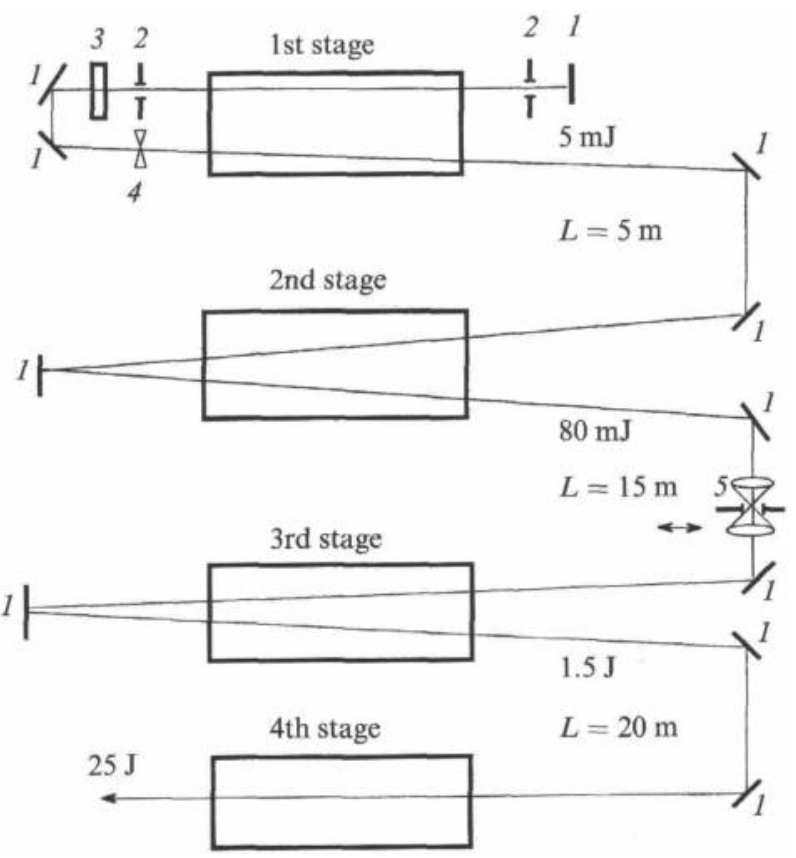

Figure 4. Optical scheme of the experiment: 1 and 4 totally reflecting mirrors, 2 pinhole with a diameter of $1.5 \mathrm{~mm}, 3$ semitransparent mirror, 5 negative lens with $F=-80 \mathrm{~cm}, 6$ telescope consisting of lenses with focal lengths of 50 and $100 \mathrm{~cm}$ ( $\mathrm{L}$ is the optical path length in between the stages). 


\begin{tabular}{lllll}
\hline Parameters & First stage & Second stage & Third stage & Fourth stage \\
\hline $\mathrm{A}, \mathrm{cm}$ & $\varnothing=0.6$ & $1.2 \times 1.8$ & $5 \times 6$ & $21 \times 25$ \\
$\mathrm{E}_{\text {in }^{\prime}} \mathrm{J}$ & - & 0.003 & 0.03 & 1.2 \\
$\mathrm{E}_{\text {out' }} \mathrm{J}$ & 0.005 & 0.08 & 1.5 & 25 \\
$\mathrm{E}_{\mathrm{d}} / \mathrm{E}_{\text {out }}$ & 0.82 & 0.77 & 0.5 & - \\
\hline
\end{tabular}

Table 1. Parameters of the radiation amplified with an MELS-4 $\mathrm{k}$ laser system.

or an increase in the diameter of the beam amplified in it leads to a decrease in the energy concentrated inside the angle $Q_{d}$ to a level of no greater than $50 \%$. Using single-pass amplification in the passive part of the active medium of the first stage, we additionally increase the output energy of the master oscillator by a factor of about 20 .

For the second stage, an input energy of $3 \mathrm{~mJ}$ is sufficient for the saturation of the amplifier active medium at two passes. A minor increase in the divergence after the second amplification stage in comparison to the divergence after the first stage is related to the presence of the ASE. The active medium of the preamplifier does not contribute to the observed increase in the divergence that is related to the distortions in the remaining part of the optical path (air and optical elements).

The atmospheric turbulent flows impede the measurements of the divergence at the output of the main amplifier. In particular, the position and structure of the focal spot are unstable when the radiation of the master oscillator passes through the optical system in the absence of amplification. The instability strongly depends on the presence of heat sources in the vicinity of the optical path and on the time interval after the operation of the amplifiers. This is the reason for the approximate value of the ratio $E_{d} / E_{\text {out }}$ for a beam size of $10 \times 12 \mathrm{~cm}$. We may state that, in this case, nearly $50 \%$ of the energy is concentrated in an angle of $5 \times 10^{-5} \mathrm{rad}$. This result is in agreement with the results of the alternative measurements in which the output radiation is focused by a lens with $F=1.5 \mathrm{~m}$ on the titanium foil with a thickness of $50 \mu \mathrm{m}$ : a hole diameter of $100 \mu \mathrm{m}$ corresponds to a divergence of about $6.5 \times 10^{-5} \mathrm{rad}$.

To more thoroughly study the effect of the heterogeneities in the active medium of the main amplifier and the optical path, we amplify the radiation of the master oscillator at three beam diameters: 35,75 , and $150 \mathrm{~mm}$, respectively. Figure 5 demonstrates the intensity distributions of the original radiation (the amplifier is switched off) and the amplified radiation measured in the far-field region. The first three panels correspond to the single-pass amplification in the active medium. It is seen that, for beam diameters of 35 and $75 \mathrm{~mm}$, the divergence of the original and amplified radiation is close to the diffraction-limited divergence $Q_{d}$. At a beam diameter of $150 \mathrm{~mm}$, the focal spot is broken into a few spots, so that the divergence is significantly higher than $Q_{d}$.

The most probable reason for this lies in the fluctuations of the air density in the optical path, since, in the case under consideration, the distance between lens 5 and the focal spot is about $25 \mathrm{~m}$. We change the optical scheme to decrease the possible effect of air. In the new scheme, the amplified radiation is expanded with a telescope in front of the amplifier, amplified, reflected by the mirror, amplified on the return pass, compressed by the same telescope, and detected. For detection, the reflection mirror was slightly misaligned relative to the optical axis 


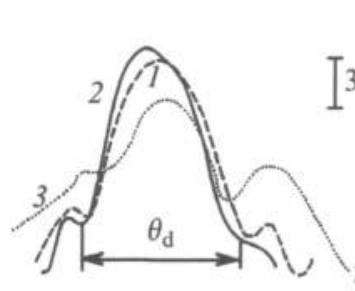

a

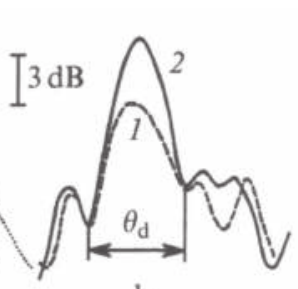

b

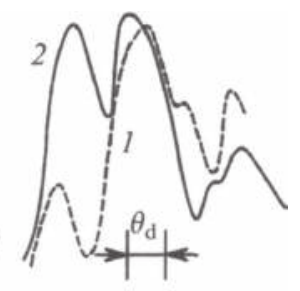

c

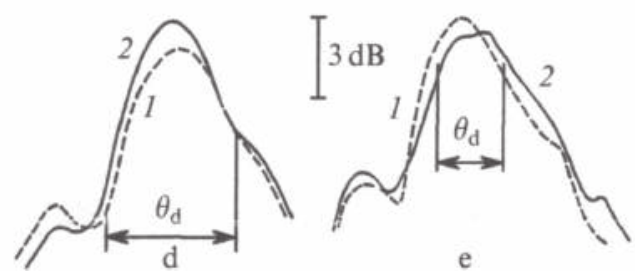

Figure 5. Densitograms of the focal spots for beam diameters of (a) 35, (b) and (c) 75, and (d) and (e) 150 mm: 1 original radiation, 2 amplified radiation, and 3 original radiation having passed through the amplifier a few seconds after its work.

of the system. Figure $\mathbf{5 d}$ and $\mathbf{e}$ shows the densitograms of the focal spots for the new scheme. It is seen that the focal spot remains unbroken even at a beam diameter of $150 \mathrm{~mm}$. The calculations show that a certain broadening in comparison to the diffraction size is related to the spherical aberration in the telescope.

In the measurements of the densitograms, the time interval between the amplifier activations is no less than $30 \mathrm{~min}$. At shorter pauses, the divergence of the original radiation increases even for a beam diameter of $35 \mathrm{~mm}$ (Figure 5a). Nevertheless, the distortions are not observed when the radiation of the master laser enters the working medium of the amplifier immediately after the termination of the pump pulse.

\section{Laser system with an output aperture of $40 \mathrm{~cm}$}

The laser system consists of five excimer lasers (Photon-1-Photon-5), the synchronization and starting system, and the matching optical elements. In three lasers, the working mixture is excited by an electric discharge. In two lasers, electron-beam excitation is used. In the experiments, the parameters of the laser radiation are measured using conventional methods and devices. To measure the time and energy characteristics of the laser pulses, we employ an FEK-22 vacuum photodiode, TPI and IKT-2 N calorimeters, and an OPHIR calorimeter with an L30A-EX head. The signals are detected with Tektronix oscilloscopes.

\subsection{Discharge lasers}

Figure 6 shows the exterior view of the first laser (Photon-1) [34]. The electrodes of the discharge gap are mounted inside the steel discharge chamber of the laser, which has a diameter 


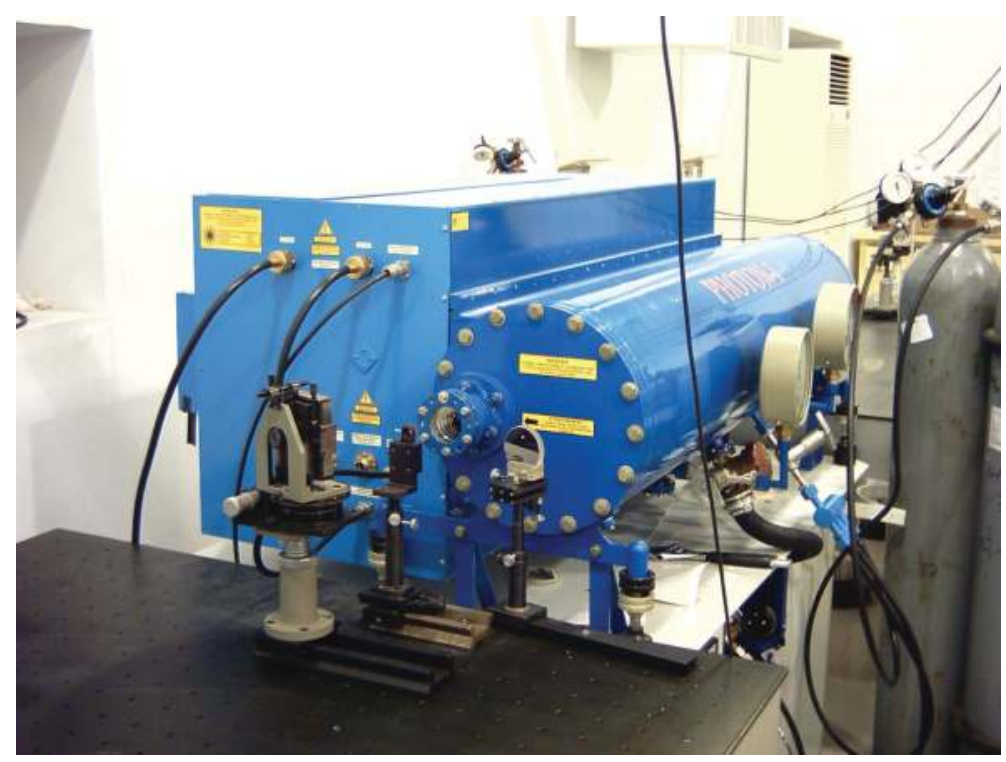

Figure 6. Photograph of the Photon-1 laser setup.

of $35 \mathrm{~cm}$. The electrode length is $107 \mathrm{~cm}$, its active length is $102 \mathrm{~cm}$, and the width of the interelectrode gap is $4 \mathrm{~cm}$. A rectangular hole on one of the sides of the laser chamber is covered with an insulator. The elements of the system for the laser excitation are placed on the insulator surface and are covered with a metal housing.

For the excitation, we employ an electric circuit with inductive energy storage and a semiconductor current breaker [35]. In this circuit, the high-voltage pulse with a short leading edge that is needed for discharge ignition is formed with a peaking capacitor $C=3.2 \mathrm{nF}$ that is charged by the inductive energy storage with 12 SOS diodes [36]. The discharge preionization is realized upon the charging of the peaking capacitors owing to the radiation of 90 spark gaps that are uniformly distributed on the two sides of the anode. The main energy is deposited to the active medium at a low optimal voltage supplied by the storage capacitor $(550 \mathrm{nF})$ that consists of K15-10 ceramic capacitors. The laser cavity consists of dielectric mirrors with reflectances of $98 \%$ and $7 \%$. For the mixture $\mathrm{Ne}: \mathrm{Xe}: \mathrm{HCl}=1520: 10: 1$ with a pressure of $2 \mathrm{~atm}$, the maximum laser energy is higher than $1.5 \mathrm{~J}$ at a pulse duration of $300 \mathrm{~ns}$ (FWHM) and a total efficiency of $1.35 \%$. The low efficiency was due to the low content of $\mathrm{HCl}$ in the mixture, which was necessary to ignite the bulk discharge with large duration ( $500 \mathrm{~ns})$.

The two remaining electric-discharge lasers (Photon-2 and Photon-3) [37, 38] are virtually identical with respect to the design and the pumping scheme. Figure 7 demonstrates the exterior view of Photon-3. Each of these lasers consists of three units that contain the gas-discharge chamber with the pump generator and electric and pneumatic control panels. The chamber contains the vacuum diode of the soft $x$-ray source, the electrodes of the laser discharge gap, and the insulator of the high-voltage input. The electrodes are made of stainless steel. For the Photon-2 (Photon-3) laser, the interelectrode distance is 5.4 (9) $\mathrm{cm}$ and the electrode length 


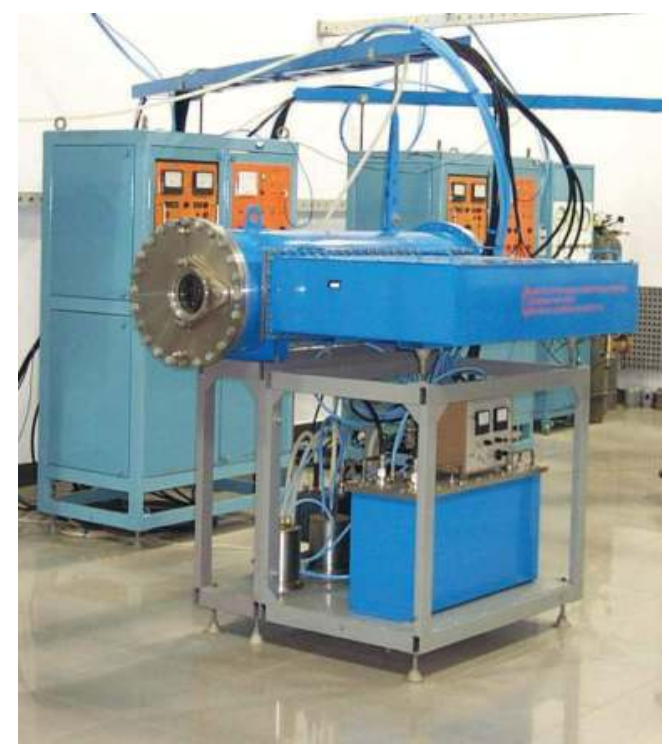

Figure 7. Photograph of the Photon-3 laser setup.

is $80(100) \mathrm{cm}$. The $\mathrm{x}$-ray radiation is transmitted to the discharge gap through the electrode window, which is covered with $80-\mu$ m-thick titanium foil. The anode of the discharge gap is connected to the pump generator located outside the chamber via an insulator with metal studs. The design of the elements that connect the generator to the anode and the backward wire-ways provides for the minimum inductance of the discharge circuit.

The vacuum diode of the x-ray source has a cylindrical housing that accommodates the anode and the cold cathode working upon explosive electron emission. The emission surface of the cathode consists of strips of foil-clad fiberglass plastic. The strips are attached to a separating grid that is covered with a $40-\mu \mathrm{m}$-thick titanium foil for the sealing of the vacuum diode and is used for the out coupling of the x-ray radiation. A tantalum foil serves as the anode of the vacuum diode. The vacuum diode is evacuated with an oil-diffusion pump to a residual pressure of about $10^{-4}$ torr.

A three-stage Arkad'ev-Marx generator with a fast capacitance of $15 \mathrm{nF}$ serves as the power supply for the vacuum diode. The generator is connected to the vacuum diode with a KVI-120 high-voltage cable. A positive voltage pulse with an amplitude of $50-55 \mathrm{kV}$ and a duration of $700 \mathrm{~ns}$ is fed to the anode. The $\mathrm{x}$-ray doze in the cathode region of the discharge gap is $20-30 \mathrm{mR}$.

The storage capacitor, the switching unit, and the peaking capacitor are the main elements of the laser pump generator. The storage capacitor $C_{L}$ consists of two (Photon-2) or three (Photon-3) FL-300 lines connected in parallel. The electric length of the line is $300 \mathrm{~ns}$, its capacitance is $150 \mathrm{nF}$, and the impedance is $1 \Omega$. The pulsed charging of the line employs an IK-100 capacitor that is connected to the line with a KVI-120 cable. This capacitor can be charged to a voltage of $40-65 \mathrm{kV}$. The peaking capacitors $\mathrm{C}_{2}=4.9$ (Photon-2) and $6.9 \mathrm{nF}$ (Photon-3) that form the space discharge in the gap represent the batteries of KVI-3 ceramic capacitors (20 kV and $680 \mathrm{pF}$ ). 
A rail-gap switch exhibits a low inductance. Its electrodes are made of stainless steel. For the Photon-2 (Photon-3) laser, the electrode length is 80 (100) cm and the interelectrode distance is 4 (6) $\mathrm{mm}$. A starting electrode made up of foil leaves is located in the vicinity of the cathode along its entire length. The gaps are activated when a high-voltage pulse is fed to the starting electrode. The gap case represents a dielectric tube with an outer diameter of $65 \mathrm{~mm}$. In the working mode, the gaps are filled with dry air at a pressure of 4-6.6 atm.

The high-voltage pulse needed for the activation of the spark gaps is produced by a high-voltage impulse generator based on a TGI-1-1000/25 thyratron. The artificial delay lines of the synchronization system provide for a sequential switching on of the pump generator and the x-ray source.

Plane-parallel plates made of fused quartz serve as the windows of the laser chamber. In the lasing mode, the laser cavity is formed by the external dielectric mirror with a reflectance of $98 \%$ at a wavelength of $308 \mathrm{~nm}$ and the window of the laser chamber. The laser mixture has a pressure of 3.5-4 atm and consists of the gases $\mathrm{Ne}, \mathrm{Xe}$, and $\mathrm{HCl}$.

The free-running Photon-2 and Photon-3 lasers generate pulses with a duration of 250-300 ns (FWHM) and an energy of 3.5 and $10 \mathrm{~J}$, respectively.

\subsection{Electron beam-pumped lasers}

Figure 8 shows a photograph of the forth laser (Photon-4). The laser consists of the Arkad'ev-Marx generator (high-voltage generator), vacuum diode, laser chamber, gas-filling and evacuation system, and electric control panel. The vacuum diode and the generator that serves as its power supply are placed in a single case. Thus, vacuum insulation of the high-voltage components is realized in the high-voltage generator. This design makes it possible to minimize the inductance of the power-supply circuit of the vacuum diode, as well as the accelerator size and weight. The high-voltage generator has three parallel branches that make it possible to minimize the inductance and the erosion of the gap electrodes. In each branch, the spark-gap space is filled with dry air mixed with $\mathrm{SF}_{6}$. The capacitance of each stage in one branch is $0.18 \mathrm{nF}$. The accelerator is started with a controlled gap switched to a high-voltage cable connected to the high-voltage generator. The gap is activated by a high-voltage pulse of the thyratron generator. The vacuum-diode cathodes with a total length of $110 \mathrm{~cm}$ are mounted on a holder fixed on the upper stage of the high-voltage generator. The laser chamber that serves as the anode is located in between the cathodes in the center of the vacuum diode. The inner diameter of the laser chamber is $25 \mathrm{~cm}$. At the entire length, the chamber is attached to the diode housing with a metal plate. The plate facilitates the current flow and decreases the electron beam loss owing to the effect of the self-magnetic field. In the absence of the plate, when the current is closed only on the ends of the gas chamber, the loss may amount to $50 \%$. Velvet serves as the electron emitter on the cathodes of the vacuum diode. The width of the anode-cathode gap is $7 \mathrm{~cm}$. Thus, four radially converging beams formed in the diode are injected into the laser chamber through eight windows (two windows in a row with a total length of $120 \mathrm{~cm}$ ). Each vacuum-sealed window is closed with a $40-\mu \mathrm{m}$-thick titanium foil attached to a metal grid. The residual gas pressure in the case that accommodates the high-voltage generator and the vacuum diode is $5 \times 10^{-5}$ torr. 


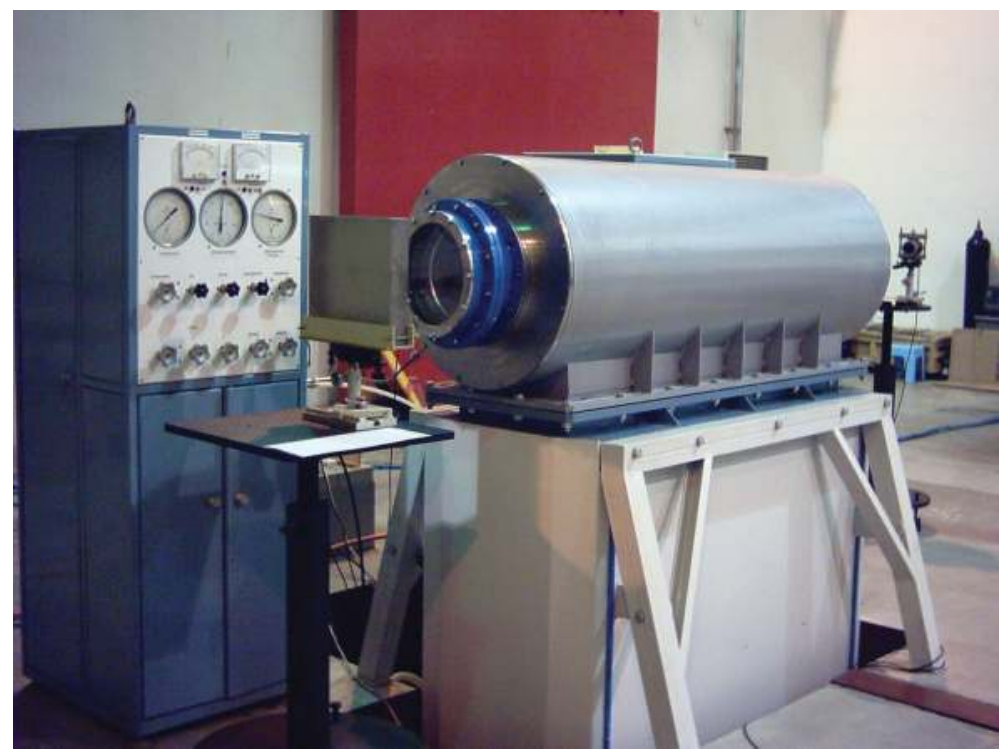

Figure 8. Photograph of the Photon-4 laser setup.

At a charging voltage of $85 \mathrm{kV}$, the voltage pulse formed by the generator across the diode has a duration of $1000 \mathrm{~ns}$, an amplitude of $480 \mathrm{kV}$, and a total current of $74 \mathrm{kA}$. The electron beam generated in the diode provides for a relatively homogeneous excitation of the laser mixture.

The plane-parallel plates, which have a diameter of $300 \mathrm{~mm}$ and are made of fused quartz, serve as the windows of the laser chamber. In the lasing mode, the laser cavity is formed by the Al-coated external mirror and the window of the laser chamber.

The laser mixture consists of argon, xenon, and $\mathrm{HCl}$. For the mixture Ar: $\mathrm{Xe}: \mathrm{HCl}=760: 20: 1$ with a pressure of $2 \mathrm{~atm}$, the radiation pulse energy amounts to $120 \mathrm{~J}$ at a charging voltage of $85 \mathrm{kV}$. The FWHM of the laser pulse is about 250 ns. Figure 9 shows the exterior view of the Photon-5 laser. The laser gas mixture is excited by a radially converging electron beam from six directions [8]. The beam is generated in a vacuum diode that contains 18 cathodes. The cathode profile is chosen in accordance with the results of the numerical calculations of the beam parameters using the original 2D code. The emitting cathode surface is made of carbotextim (graphite-fiber material with a resistivity of about [5-50] $\times 10^{-2} \Omega \mathrm{m}$ ), and it is covered with velvet. The width of the emitting surface is $120 \mathrm{~mm}$ and the total area of the cathode-emitting surface is $0.95 \mathrm{~m}^{2}$. The interelectrode gap between the emitting surface and the supporting structure of the outcoupling window is $6 \mathrm{~cm}$. The supporting structure contains 18 windows (three windows in a row with a total length of $150 \mathrm{~cm}$ ). The geometrical transparency of the beam outcoupling system is about $75 \%$. The beam is outcoupled to the laser chamber through the Ti foil with a thickness of $40 \mu \mathrm{m}$. The laser cell diameter is $41 \mathrm{~cm}$ and the cell volume is 2001 .

The voltage pulse across the diode is generated using two paralleled linear transformers with the vacuum insulation of the secondary coil. Each of the transformers consists of ten stages, 


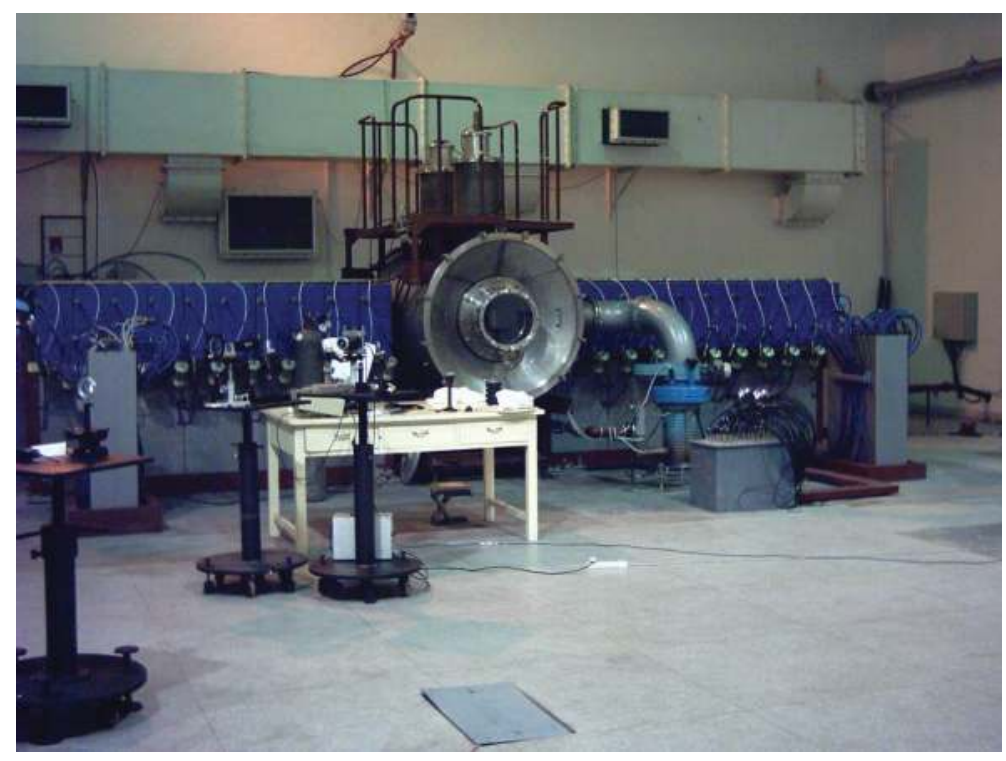

Figure 9. Photograph of the Photon-5 laser setup.

and each of the stages consists of eight IK-100-0.17 capacitors $(100 \mathrm{kV}, 17 \mu \mathrm{F}$, and $50 \mathrm{nH})$. The output power of the stage is about $12 \mathrm{GW}$. The voltage is fed to the diode (the cathode collector) with vacuum lines that serve as the secondary coils of the transformer. The diameter of the diode vacuum chamber is $131 \mathrm{~cm}$ and its length is $210 \mathrm{~cm}$. The collector is suspended coaxially to the vacuum chamber on two springs in the upper part of the chamber. The vacuum chamber is evacuated using two AVDM-250 vacuum devices with liquid-nitrogen traps to a residual pressure in the diode of $(3-4) \times 10^{-5}$ torr.

At a charging voltage of $85 \mathrm{kV}$, the amplitude of the voltage pulse across the vacuum diode amounts to $550 \mathrm{kV}$. In this case, the total current (the sum of two currents) is $320 \mathrm{kA}$, and the energy transferred from the transformer to the diode is $87 \mathrm{~kJ}$. At a charging voltage of $80 \mathrm{kV}$, the voltage across the vacuum diode is $440 \mathrm{kV}$, the total current is $290 \mathrm{kA}$, and the energy transferred to the diode is $78 \mathrm{~kJ}$. The energy input to the gas increases when the pressure increases to $2.5 \mathrm{~atm}$. Then, the energy input remains virtually unchanged when the pressure increases to $3.5 \mathrm{~atm}$. The maximum energy input to the gas from the electron beam is about $19 \mathrm{~kJ}$. The efficiency of the energy transfer from the primary storage to the gas is about $19 \%$. This result is close to the value realized in conventional accelerators with water lines [1-3].

Plane-parallel plates with a diameter of $400 \mathrm{~mm}$ made of fused quartz serve as the windows of the laser chamber. This laser is tested in lasing mode with the cavity formed by the Al-coated plane mirror and the laser chamber window. For the mixture $\mathrm{Ar}: \mathrm{Xe}: \mathrm{HCl}=760: 20: 1$ with a pressure of $2 \mathrm{~atm}$, the radiation pulse energy amounts to $660 \mathrm{~J}$ at a charging voltage of $85 \mathrm{kV}$ [8]. The radiation pulse FWHM is about $350 \mathrm{~ns}$. The heterogeneity of the radiation energy density distribution over the cross section of the laser beam is less than $10 \%$. 


\subsection{Laser system}

Figure 10 demonstrates the block diagram of the synchronization and starting system that is used to synchronize the setups. The laser system is controlled with a PC that is interfaced with a synchronization pulse generator producing voltage pulses with an amplitude of $600 \mathrm{~V}$ and a variable interpulse delay. These pulses are used to start four thyratron generators and the magneticbias generator of the Photon-5 laser. The last generator produces two bias pulses that are fed to the linear transformers to premagnetize the transformer cores in the appropriate direction. The thyratron generators produce negative voltage pulses with an amplitude of about $20 \mathrm{kV}$. These pulses are fed to the spark gaps of the Photon-1, Photon-2, and Photon-3 lasers and to the inputs of the generators of the Photon- 4 and Photon- 5 lasers. Both generators produce negative pulses with an amplitude of about $85 \mathrm{kV}$, which are used to trigger the rail gaps of the Photon-2 and Photon-3 lasers and to start the Photon- 4 laser. These pulses are also fed to the inputs of the trigger generators G-1 and G-2. The trigger generators produce 40 negative pulses with an amplitude of $85 \mathrm{kV}$ that activate the gaps of the transformer stages of the Photon- 5 laser. In addition, the PC controls the on-off switching of the capacitive storage charging in all of the lasers with allowance for the particular charging times, so that the storages are charged simultaneously.

Thus, at the first stage of the laser system's operation, the storage capacitors of all of the lasers are charged. Then, the starting pulse is fed to the magnetic-bias generator of the Photon-5 laser and a certain sequence of the starting pulses is fed to the thyratron generators of the Photon-1, Photon-2, Photon-3, and Photon-4 lasers. Thus, we activate the system as a whole.

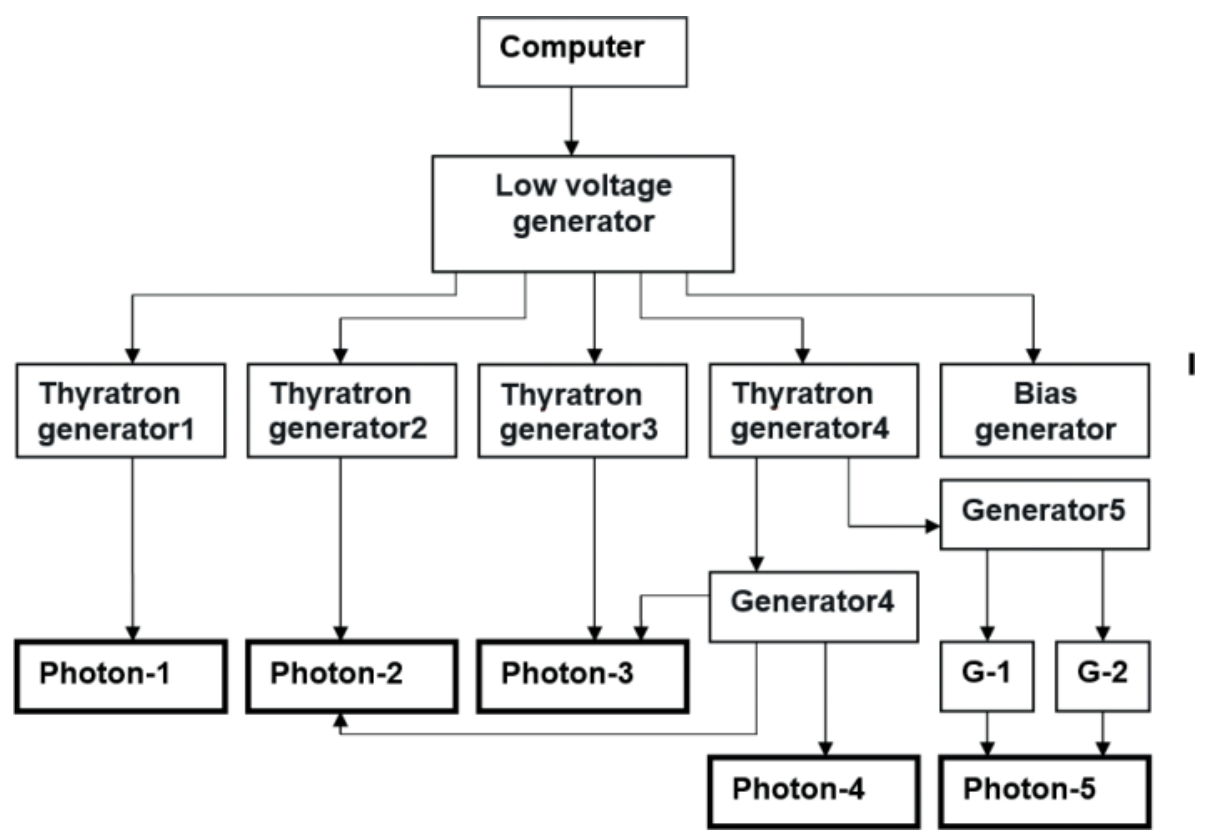

Figure 10. Block diagram of the starting and synchronization system. 
In the laser system, the Photon-2, Photon-3, Photon-4, and Photon-5 lasers start working as slave amplifiers. In this case, the windows of all of the laser chambers are tilted at angles providing for the absence of feedback. The Photon-1 laser serves as the master oscillator [34]. Its optical scheme makes it possible to generate a high-quality beam in a certain part of the active volume and to amplify this beam in the remaining part. To improve the spatial structure of the radiation, we employ two pinholes with a diameter of $2 \mathrm{~mm}$. In this case, the Fresnel number is $N \sim 2$ for a cavity with a length of $1.5 \mathrm{~m}$. The spectral selection is realized with an auto-collimation diffraction grating $\left(1800 \mathrm{~mm}^{-1}\right)$. The feedback in the cavity is maintained via the first diffraction order. To decrease the contribution of the noise component to the output radiation, we outcouple the laser beam through a semitransparent mirror with a reflectance of $R=30 \%$. Then, the low-power high-quality radiation of the master laser is additionally amplified at two passes in the same active medium, so that the output beam diameter increases to $7 \mathrm{~mm}$. The output pulse of the Photon- 1 laser has an energy of $50 \mathrm{~mJ}$, a duration of $250 \mathrm{~ns}$, and a spectral line width of $0.9 \mathrm{~cm}^{-1}$. The divergence of the laser beam, which contains $80 \%$ of the energy $(0.13 \mathrm{mrad})$, is greater than the diffraction-limited divergence by a factor of 1.2 .

This beam is expanded using a lens telescope with a magnification of $M=1.5$ to match the beam diameter with the sizes of the active media of the Photon- 2 and Photon- 3 lasers. The beam is amplified at three passes in the Photon- 2 laser and one pass in the Photon- 3 laser. The output beam diameters of these lasers are 3 and $6 \mathrm{~cm}$, respectively. For further matching of the beam diameter with the sizes of the active media of the Photon- 4 and Photon- 5 lasers, we employ a lens telescope with a fivefold magnification. After the beam expansion, the radiation is amplified at one pass in the active medium of the Photon-4 laser and at one or two passes in the active medium of the Photon-5 laser.

Table 2 summarizes the experimental results for the laser system. The maximum energy (330 J) is obtained in the case of the single-pass amplification in the Photon-5 laser when the ASE flux and the absorption in the active medium are minimized. In the case of double pass amplification, both the absorption and the ASE contribution increase (the ASE intensity increases owing to the reflection from the rear mirror with $R=99 \%$ ). This leads to a decrease in the energy of the amplified radiation to a level of $250 \mathrm{~J}$.

\begin{tabular}{|c|c|c|c|c|c|}
\hline Lasers & Operating mode & Energy, $\mathrm{J}$ & Pulse duration, ns & Line width, $\mathrm{cm}^{-1}$ & Divergence, $\mu \mathrm{rad}$ \\
\hline Photon-1 & $\begin{array}{l}\text { Laser } \\
\text { master } \\
\text { oscillator }\end{array}$ & $\begin{array}{l}1.5 \\
0.05\end{array}$ & $\begin{array}{l}300 \\
200-250\end{array}$ & $\overline{-}-9$ & $\begin{array}{l}- \\
130\end{array}$ \\
\hline Photon-2 & $\begin{array}{l}\text { Laser } \\
\text { amplifier }\end{array}$ & $\begin{array}{l}3.5 \\
0.5\end{array}$ & $\begin{array}{l}250 \\
200-250\end{array}$ & $\overline{-} .9$ & $\begin{array}{l}- \\
60\end{array}$ \\
\hline Photon-3 & $\begin{array}{l}\text { Laser } \\
\text { amplifier }\end{array}$ & $\begin{array}{l}10 \\
5\end{array}$ & $\begin{array}{l}300 \\
200-250\end{array}$ & $\overline{-} .9$ & $\begin{array}{l}- \\
37\end{array}$ \\
\hline Photon-4 & $\begin{array}{l}\text { Laser } \\
\text { amplifier }\end{array}$ & $\begin{array}{l}120 \\
40\end{array}$ & $\begin{array}{l}250 \\
200-250\end{array}$ & - & $\begin{array}{l}- \\
-\end{array}$ \\
\hline Photon-5 & $\begin{array}{l}\text { Laser } \\
\text { amplifier }\end{array}$ & $\begin{array}{l}660 \\
250,330\end{array}$ & $\begin{array}{l}350 \\
200-250\end{array}$ & - & - \\
\hline
\end{tabular}

Table 2. Parameters of the radiation of the photon lasers. 


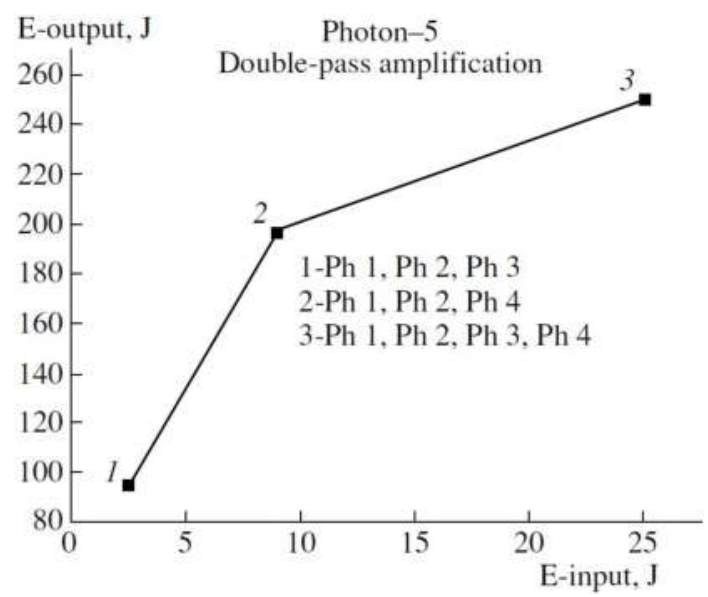

Figure 11. Plot of the output radiation energy vs. the input signal energy for the double-pass amplification in the active medium of the Photon-5 laser.

The spectral and spatial parameters of the radiation are measured only for the first three setups (Photon-1, Photon-2, and Photon-3). The divergence is measured using the spot size in the focal plane of a lens with a focal length of $F=13.5 \mathrm{~m}$. The line width is measured with the Fabry-Perot interferometer. In both cases, the radiation intensity distribution is detected by a CCD array. The spectral measurements show that the line width remains unchanged $\left(0.9 \mathrm{~cm}^{-1}\right)$ after the amplification. In general, the divergence of the amplified radiation decreases with an increase in the beam diameter. However, the divergence is slightly greater than the diffraction-limited divergence due to the distortions in the active medium and the optical path.

Figure 11 shows the dependence of the output energy of the main amplifier on the input energy upon the double-pass amplification. The input energy is measured when one of the amplifiers (Photon-3 or Photon-4) is switched off. It is seen that the Photon-5 amplifier is saturated only when all of the amplifiers are switched on. In this case, the gain is about 10 . The laser beam spot on photosensitive paper exhibits a relatively homogeneous distribution and a minor contribution of the diffraction rings related to the heterogeneities in the optical path. This indicates a high spatial coherence of the output radiation.

\section{Laser system THL-100}

\subsection{Femtosecond pulse generator}

The femtosecond pulse generator (front-end) of laser system consists of a femtosecond Ti:sapphire master oscillator, regenerative and multipass amplifiers, a pulse stretcher and a pulse compressor, and generator of second harmonic. It operates at a pulse repetition rate of $10 \mathrm{~Hz}$ and in a single pulse mode. Output energy of front-end is up to $5 \mathrm{~mJ}$ at $475 \mathrm{~nm}$. Transform-limited pulse duration is $50 \mathrm{fs}$. 


\subsection{Photodissociation excimer $\mathrm{XeF}(\mathrm{C}-\mathrm{A})$ amplifier}

$\mathrm{XeF}(\mathrm{C}-\mathrm{A})$ amplifier and its schematic cross section are shown in Figures 12 and 13, respectively. It consists of two high-voltage pulse generators, a vacuum chamber with six electron cathodes, a xenon gas chamber, and a laser cell. For multipass laser beam amplification, the laser cell has a mirror unit (32 mirrors).

Two high-voltage pulse generators are line transformers, which have twelve transformer stages. Each stage has $320 \mathrm{nF}$ capacitors and multigap spark switch. A secondary turn of the transformer is a vacuum coaxial line, which is connected with an e-beam diode. The diode has six explosive emission cathodes (flock-coated metal). The anode of vacuum diode is $45 \mathrm{~cm}$-dia gas chamber closed by $40-\mu \mathrm{m}$-thick titanium foil (Figure 14). The vacuum diode forms six radially converging electron beams, each of $15 \times 100 \mathrm{~cm}^{2}$ area, that are injected into gas chamber filled by xenon at a $3 \mathrm{~atm}$ pressure. The capacitors of each stage can be charged up to $90-100 \mathrm{kV}$.

At charge voltage of $95 \mathrm{kV}$, the energy stored in the capacitor of a line transformer is $34.6 \mathrm{~kJ}$, the energy of electron beam in the vacuum diode is $21 \mathrm{~kJ}$, and the energy delivered to the gas chamber is $7 \mathrm{~kJ}$. E-beam in the vacuum diode has following parameters: peak voltage of $550 \mathrm{kV}$, peak current of $200 \mathrm{kA}$, and current pulse duration of $150 \mathrm{~ns}$ (FWHM). The electron beam is injected into the gas chamber and one almost completely is absorbed by xenon at its thickness of $8 \mathrm{~cm}$. An efficiency of the electron beam energy converting to VUV radiation of $\mathrm{Xe}_{2}{ }^{*}$ excimers is $30-40 \%$ [27].

The laser cell (Figure 15) is housed into the gas chamber along its axis and contains a gas mixture of $\mathrm{XeF}_{2}$ and high-purity nitrogen at partial pressures of $0.2-0.4$ and 190-380 torr, respectively. The active medium of amplifier on $\mathrm{XeF}(\mathrm{C}-\mathrm{A})$ molecules is excited by the VUV radiation $(172 \mathrm{~nm})$ due to photodissociation of $\mathrm{XeF}_{2}$ molecules with the $\mathrm{XeF}(\mathrm{B})$ molecules formation. The

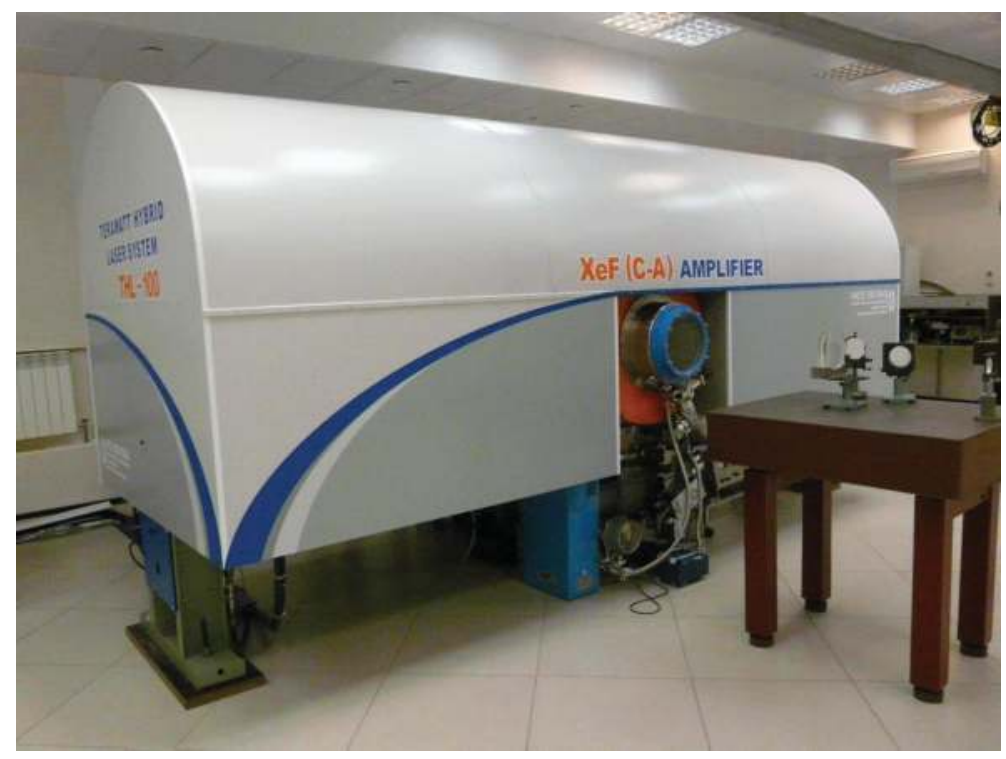

Figure 12. Photo of the $\mathrm{XeF}(\mathrm{C}-\mathrm{A})$ amplifier. 


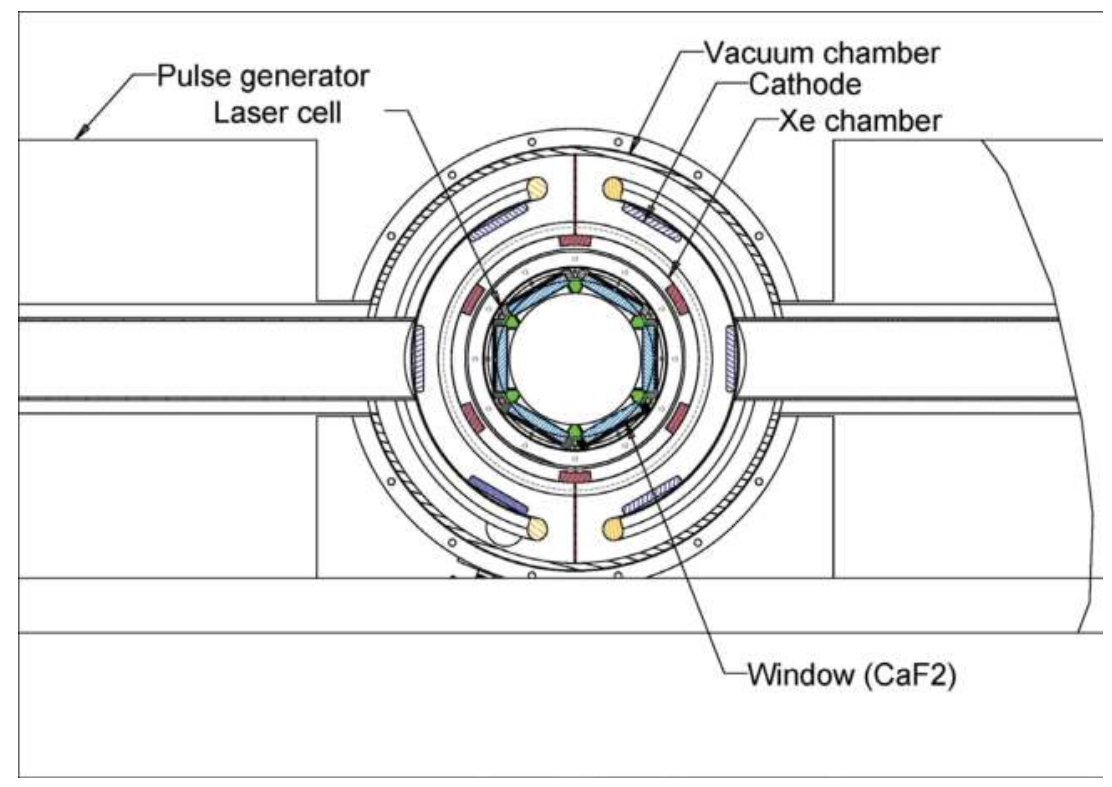

Figure 13. Schematic of the pump circuit of the $\mathrm{XeF}(\mathrm{C}-\mathrm{A})$ amplifier active medium.

$\mathrm{XeF}(\mathrm{C})$ state of the laser $(\mathrm{C}-\mathrm{A})$ transition is formed due to relaxation of the $\mathrm{XeF}(\mathrm{B})$ molecules in collisions with buffer gas of $\mathrm{N}_{2}$. The active medium of $110 \mathrm{~cm}$ long is pumped through arrays of $54 \mathrm{CaF}_{2}$ windows (square of $12 \times 12 \mathrm{~cm}^{2}$ ) located on the side walls of the hexahedral laser cell against the foils through which the electron beams are injected into the gas chamber. This ensures the highest geometric coupling of the pumping source with the active medium. The windows are vacuum sealed by Viton gaskets. The hexagon laser cell has a clear aperture of $25 \mathrm{~cm}$. The laser cells are sealed by the end flanges with $30 \mathrm{~cm}$-dia fused silica windows.

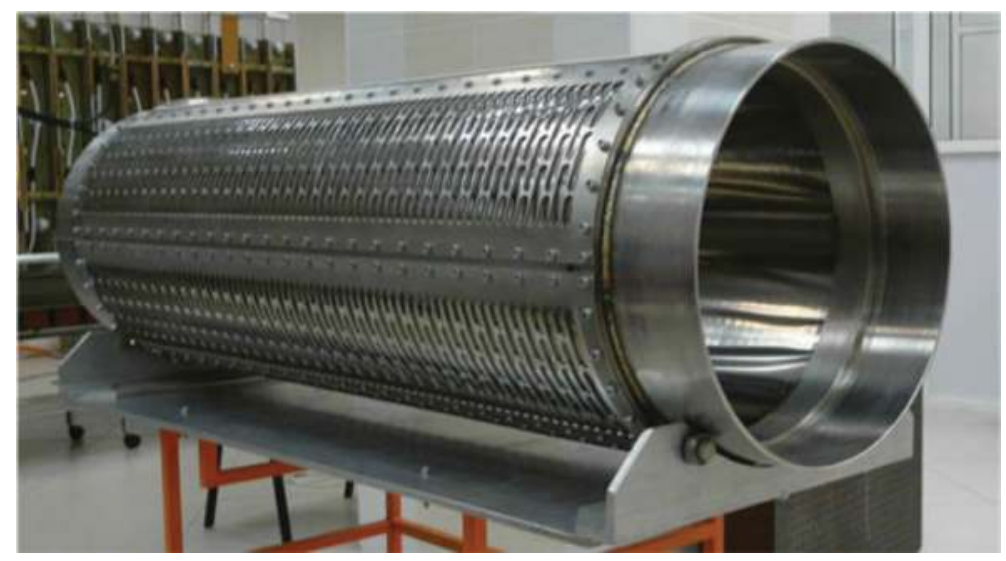

Figure 14. Gas chamber of the $\mathrm{XeF}(\mathrm{C}-\mathrm{A})$ amplifier with windows for electron beam injection. 


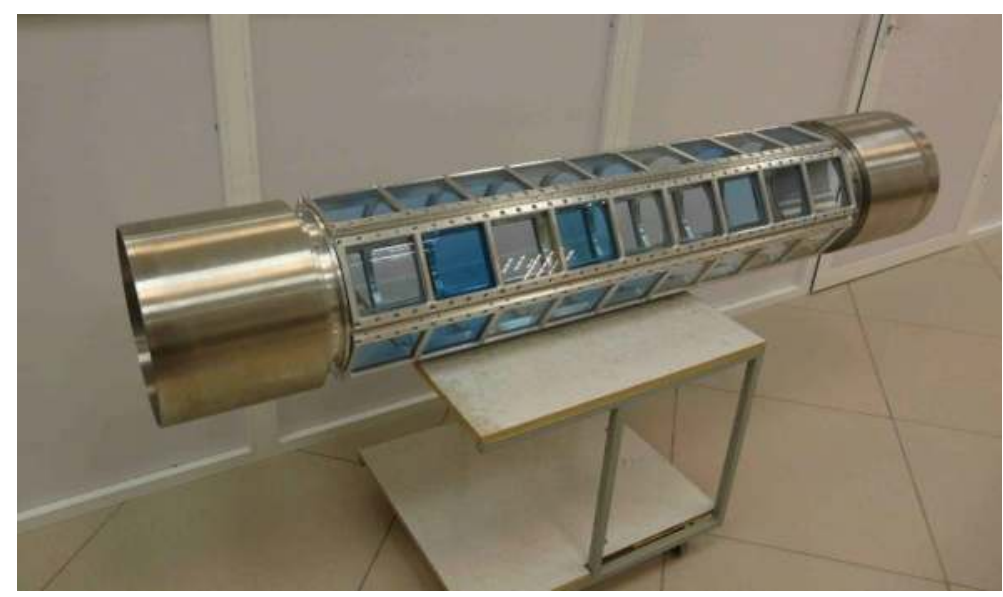

Figure 15. Laser cell of the $\mathrm{XeF}(\mathrm{C}-\mathrm{A})$ amplifier.

A high-purity xenon (99.9997\%) was supplied to the gas chamber to provide maximum efficiency of the e-beam to VUV radiation conversion. The chamber preliminary evacuated to a pressure of $10^{-4}$ torr. During operation of the amplifier, the intensity of xenon VUV radiation gradually decreased due to outgassing from the stainless steel walls of the gas chamber, exposed to the electron beam. Xenon was circulated through a Sircal MP-2000 purifier to support the gas purity recovery.

In our experiments, the mixture in the laser cell was replaced after each shot, because repeated pumping of the mixture decreased the output energy by $20-30 \%$.

\subsection{Measurement procedures}

The laser beam of front-end was amplified in the $\mathrm{XeF}(\mathrm{C}-\mathrm{A})$ amplifier in a multipass optical scheme (33 passes) formed by 32 round mirrors increasing in diameter. The mirrors with a reflectivity of $99.7 \%$ were fixed along the perimeter of the intracell flanges of the laser cell. A divergent laser beam is injected into the laser cell, so that it expands in diameter from $2 \mathrm{~cm}$ at the inlet to $6 \mathrm{~cm}$ after 32 passes. When traveling between the mirrors, the beam makes two circular rounds in the laser cell. The penultimate convex mirror directs the beam to a flat $10 \mathrm{~cm}$ diameter mirror located on the optical axis of the laser cell. A reflected beam propagates along the optical axis and is ejected from the laser cell with a diameter of $12 \mathrm{~cm}$.

Input in the $\mathrm{XeF}(\mathrm{C}-\mathrm{A})$ amplifier laser pulse was preliminary stretched up to 1 ps in a prism stretcher with negative group-velocity dispersion. The amplified negatively chirped laser pulse was expanded up to $20 \mathrm{~cm}$ and then one was collimated and compressed in three fused silica plates of $4 \mathrm{~cm}$ thickness at a Brewster angle on two passages. The energy loss in the compressor was about $2 \%$. For measurement of the amplified pulse duration, the $90 \mathrm{~mm}$-dia central part of the beam was attenuated by two wedges and focused by a 12-m focal length spherical mirror into a $0.25 \mathrm{~mm}$-dia aperture placed in front of an ASF-20-480 single-shot autocorrelator. The sech ${ }^{2}$ function was used in autocorrelator to fit the temporal intensity profile. 
The energies of the e-beams in the converter and VUV radiation in the laser chamber were measured by TPI-2-7 calorimeter. The output energy ща $\mathrm{XeF}(\mathrm{C}-\mathrm{A})$ amplifier was measured with an OPHIR energy meter placed in the output beam, which was attenuated with a fused silica wedge and focused to a spot of $2.5 \mathrm{~cm}$. The part of the beam passed through the wedge plate was used to record an image of laser beam on a photographic paper. The small signal gain of active medium was measured using a Sapphire-488 CW semiconductor laser after four passes of its probe beam through the active medium. This laser emits at $488 \mathrm{~nm}$ wavelength coinciding with the amplification band maximum of the $\mathrm{XeF}(\mathrm{C}-\mathrm{A})$ transition.

\subsection{Experimental results}

First of all, the VUV radiation energy transmitted through the $\mathrm{CaF}_{2}$ windows into the laser cell was measured. Its value was 240-260 J. In view of the quantum efficiency of laser transition and $100 \%$ quantum yield of $\mathrm{XeF}(\mathrm{C})$ state production, the integral value of the energy stored in the active medium is $\sim 90 \mathrm{~J}$. In actuality, the actual lifetime of the $\mathrm{XeF}(\mathrm{C})$ state, which is determined by radiative decay and quenching, is much shorter than the pump pulse width. This makes the maximum current value of the energy stored on the $\mathrm{XeF}(\mathrm{C}-\mathrm{A})$ transition 10 times less than the integral value.

The time profile of the small-signal gain near the $\mathrm{CaF}_{2}$ windows is shown in Figure 16. These results correlate well with those found in the experiments on femtosecond pulse amplification. As can be seen in the Figure 16, the maximum gain is $0.004 \mathrm{~cm}^{-1}$ and the FWHM of the amplified signal is $\sim 200 \mathrm{~ns}$. The amplification of picosecond pulses was performed within the time interval of $146 \mathrm{~ns}$ (33 passes) close to the gain profile maximum.

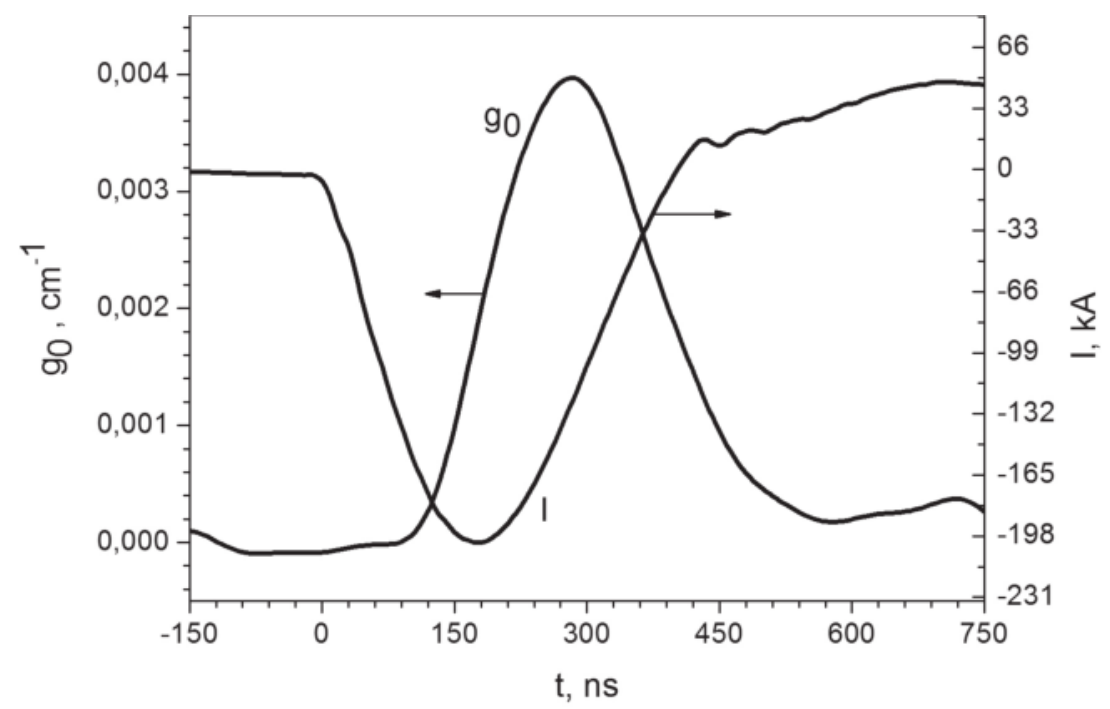

Figure 16. Time profile of the e-beam current in the diode and gain measured near the $\mathrm{CaF}_{2}$ windows with the continuous laser at $488 \mathrm{~nm}$ for a $\mathrm{XeF}_{2}$ vapor pressure of 0.25 torr. 
The small-signal gain distribution over the active medium cross section, measured at different $\mathrm{XeF}_{2}$ vapor pressures, is shown in Figure 17. These measurements show that decreasing the vapor pressure enhances the gain distribution uniformity, but at the same time, it greatly decreases the gain near the pump windows. Final optimization of the gas mixture composition was made by achieving the maximum output energy of the amplified picosecond pulse in the real multipass amplification scheme. The maximum output energy was obtained for a $\mathrm{XeF}_{2}$ vapor pressure of $0.2-0.25$ torr.

In our experiments, the seed pulse energy varied from 0.04 to $2 \mathrm{~mJ}$. At an input energy of $0.04 \mathrm{~mJ}$, the gain of active medium is far from saturation. The total gain factor was $2.5 \times 10^{3}$ by $100 \mathrm{~mJ}$ output energy. The total gain factor was reduced down to $5 \times 10^{2}$ under near-saturation conditions at a seed pulse energy of $2 \mathrm{~mJ}$. In this case, the output energy was $1 \mathrm{~J}$. The shot-to-shot fluctuations in the output pulse energy were within 10\%. An imprint of the output laser beam on a photographic paper is shown in Figure 18.

The ASE power of the $\mathrm{XeF}(\mathrm{C}-\mathrm{A})$ amplifier with seed pulse blocked was measured for an estimation of the contrast ratio. It was $1 \mathrm{~W}$ within an angle of $0.2 \mathrm{mrad}$, which is close to the output beam divergence angle. Thus, the temporal contrast ratio between the output laser peak and background ASE was $\sim 10^{13}$. It means that the real contrast of output beam is determined by the seed pulse temporal contrast, which is usually $\sim 10^{10}$ at the second harmonic frequency [39].

After compression, the pulse duration was measured both with and without amplification in active medium. In both cases, the pulse duration was 50-60 fs inferred assuming sech ${ }^{2}$ pulseshape. The output beam energy was $0.5-0.7 \mathrm{~J}$ in these experiments. The autocorrelation function for the compressed pulse at $0.7 \mathrm{~J}$ output energy, which corresponds to $50 \mathrm{fs}$ pulse duration,

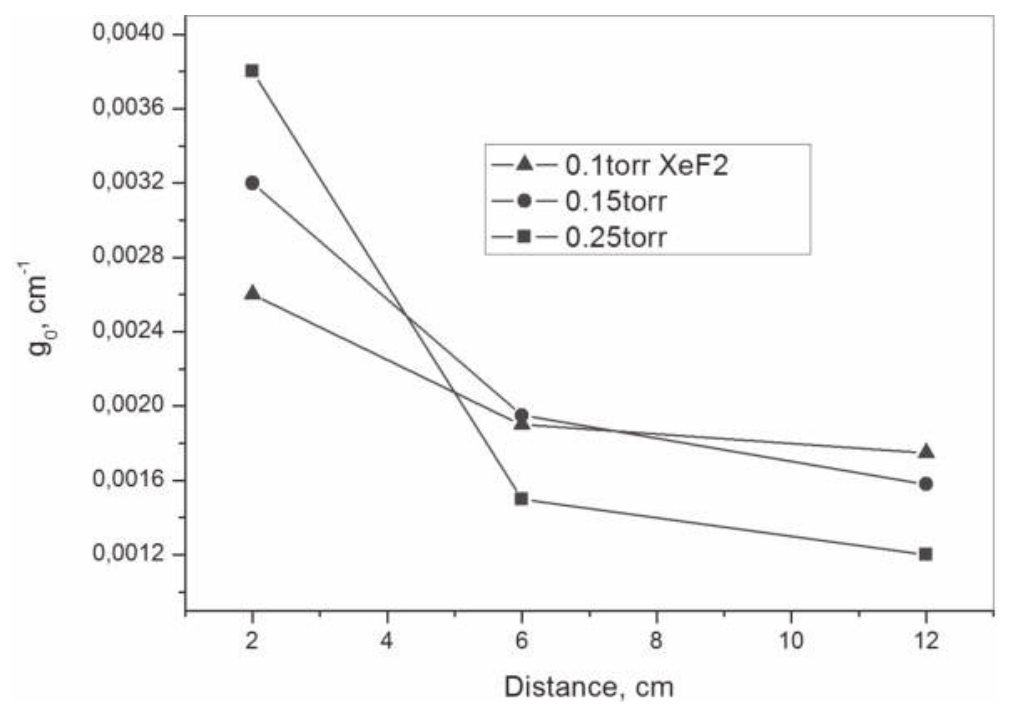

Figure 17. Small signal gain distribution from the window toward the center of the laser cell for different $\mathrm{XeF}_{2} \mathrm{vapor}$ pressures. The nitrogen pressure is 190 torr. 


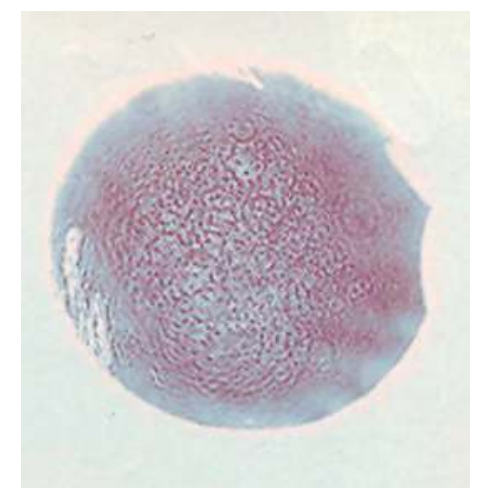

Figure 18. Autograph of the output laser beam.
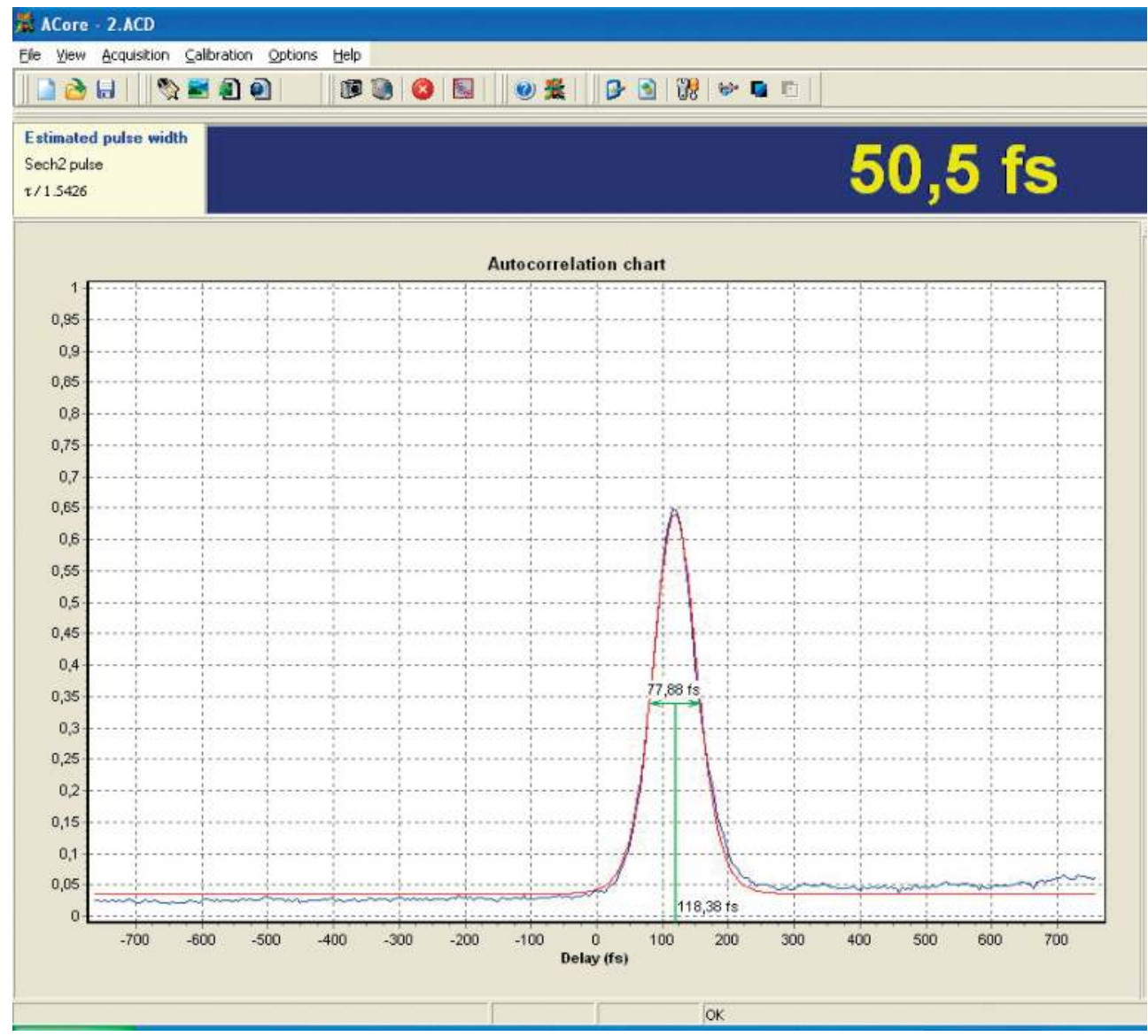

Figure 19. Pulse duration of the laser beam with energy of $0.7 \mathrm{~J}$. 
is shown in Figure 19. This result gives the peak power $~ 14$ TW. According to literature data, the highest power of femtosecond pulses in the visible spectrum was attained earlier upon nonlinear conversion to the second harmonic in a Ti:sapphire laser [39] and upon direct amplification of 250-fs pulses in an electron beam-excited $\mathrm{XeF}(\mathrm{C}-\mathrm{A})$ amplifier [40]. These values (4 TW and 1 TW, correspondingly) are far below the power attained in the present work.

\section{Conclusions}

Three wide-aperture excimer laser systems created at HCEI were presented. The schemes of small powerful lasers pumped by electric discharge and electron beam were analyzed. It was shown that electron beam-pumped lasers are more efficient for long laser pulse duration (300 ns and more). The formation results of a high-quality and high-power radiation in UV and visible range were reported.

Based on the experimental data, we demonstrated that diffraction-limited laser beams with a large cross section can be amplified in the gas laser system. It was shown that the divergence of amplified UV beam was close to the diffraction-limited beam when its diameter was no greater than $5 \mathrm{~cm}$. But the divergence increased with a further increase of diameter. The wave-front distortions on the turbulent flows in the laser mixture and air were the main reason for it. The minimum divergence of the laser beam $10 \mu \mathrm{rad}$ was obtained. The pulse energy of the amplified UV radiation at a wavelength of $308 \mathrm{~nm}$ with $250 \mathrm{~ns}$ pulse duration was $330 \mathrm{~J}$.

The output energy of $1 \mathrm{~J}$ was reached in the first pilot experiments of amplification of downchirped 1 ps pulses in femtosecond hybrid laser system THL-100. At the output energy of $0.7 \mathrm{~J}$, the recompression of the amplified pulses in bulk fused silica to the initial duration of $50 \mathrm{fs}$ was realized. This means that a record-breaking peak power $\sim 14$ TW in the visible spectrum has been attained.

\section{Author details}

Nikolay Ivanov ${ }^{1}$, Valery Losev ${ }^{1,2 *}$, Yury Panchenko ${ }^{1}$ and Viktor Tarasenko ${ }^{1,2}$

*Address all correspondence to: losev@ogl.hcei.tsc.ru

1 Institute of High Current Electronics SB RAS, Tomsk, Russia

2 National Research Tomsk Polytechnic University, Tomsk, Russia

\section{References}

[1] Obenschain SP, Bodner SE, Colombant D, Gerber K, Lehmberg RH, McLean EA, Mostivych AN, Pronko MS, Pawley CJ, Schmitt AJ, Sethian JD, Serlin V, Stamper JA, Sullivan CA. The Nike KrF laser facility: Performance and initial target experiments. Physics of Plasmas. 1996;3:2098-2107. Available from: DOI: https://doi.org/10.1063/1.871661 
[2] Owadano Y, Okudo I, Matsumoto Y, Tanimoto M, Tomie T, Koyama K, Yano M. Development of a high-power laser system, ASURA. Laser and Particle Beams. 1989;7:383-392. DOI: https://doi.org/10.1017/ S0263034600007345

[3] Dival EJ, Edwards CB, Hirst GJ, Hooker CJ, Kidd AK, Lister JMD, Mathumo R, Ross IN, Shaw MJ, Toner WT, Visser AP, Wyborn BE. Titania - A $10^{20} \mathrm{Wcm}^{-2}$ ultraviolet laser. Journal Modern Optics. 1996;43:1025-1033

[4] Bychkov YI, Ivanov NG, Losev VF, Mesyats GA. XeCl-laser with $150 \mathrm{~J}$ laser energy. Soviet Technical Physics Letters. 1988;14:253-256

[5] Abdullin EN, Gorbachev SI, Efremov AM, Koval'chuk BM, Loginov SV, Skakun VS, Tarasenko VF, Tolkachev VS, Fedenev AV, Fomin EA, Shchanin PM. High-power compact laser with $\lambda=308$ and $249 \mathrm{~nm}$ pumped by a radially converging electron beam. Quantum Electronics. 1993;23:564-567. Available from: https://doi.org/10.1070/ QE1993v023n 07ABEH 003124

[6] Ivanov NG, Losev VF, Naats EI, Ryzhov VV, Turchanovskii IY, Yastremskii AG. XeCl laser with an output energy $200 \mathrm{~J}$. Quantum Electronics. 1997;27:670-672. Available from: https://doi.org/10.1070/QE1997v027n08ABE H001029

[7] Ivanov NG, Losev VF, Panchenko YN, Yastremskii AG. XeCl laser system with a $25 \mathrm{~cm} \times 25 \mathrm{~cm}$ output aperture. Quantum Electronics. 1999;29:852-856. DOI: https:// doi.org/ 10.1070/QE1999v029n10ABEH0015 89

[8] Abdullin EN, Grishin DM, Gubanov VP, Zorin VB, Kim AA, Koval'chuk BM, Kumpyak EV, Liu J, Morozov AV, Skakun VS, Stepchenko AS, Tarasenko VF, Tolkachev VS, Tsoi NV, Shchanin PM. A 650-J XeCl laser. Quantum Electronics. 2004;34:199-202. Available from: https://doi.org/10.1070/QE2004v034n03ABEH00 2610

[9] Mesyats GA, Osipov VV, Tarasenko VF. Pulse Gas Lasers (Nauka, Moscow, 1991). Washington: Opt. Eng. Press; 1995. 272 p

[10] Kovalchuk BM, Losev VF, Mesyats GA, Tarasenko VF. Electron-beam-pumped highpower wide-aperture exciplex lasers and laser systems. Russian Physics Journal. 2000;43:352-357. DOI: 10.1007/BF025 08516

[11] Bugaev SP, Abdullin EN, Zorin VB, Koval'chuk BM, Loginov SV, Mesyats GA, Skakun VS, Tarasenko VF, Tolkachev VS, Shchanin PM. A 2-kJ wide-aperture XeCl laser. Quantum Electronics. 2004;34:801-804. DOI: https://doi.org/10.1070/QE2004v 034n09 ABEH002687

[12] Zvorykin VD, Arlantsev SV, Bakaev VG, Rantsev OV, Sergeev PB, Sychugov GV, Tserkovnikov AY. Transport of electron beams and stability of optical windows in highpower e-beam-pumped krypton fluoride lasers. Laser Particle Beams. 2001;19:609-622. DOI: $10.1017 / S 0263034601194085$

[13] Rhodes CK. Excimer Lasers. Berlin-Heidelberg: Springer-Verlag; 1984. 245 p

[14] McIntyre IA, Rhodes CK. High power ultrafast excimer lasers. Journal of Applied Physics. 1991;69:121-139. DOI: 10.1063/1.347665

[15] Watanabe M, Endoh A, Sarukura N, Watanabe S. Subpicosecond UV pulse generation for a multiterawatt KrF laser. Applied Physics B. 1989;48:417-420. DOI: 10.1007/BF00694542 
[16] Bischel WK, Eckstrom DJ, Walker HC, Tilton RA. Photolytically pumped XeF(C-A) laser studies. Journal of Applied Physics. 1981;81:4429-4434. DOI: 10.1063/1.329357

[17] Strickland D, Mourou GA. Compression of amplified chirped optical pulses. Optics Communications. 1985;56:219-221. DOI: 10.1016/0030-4018(85)90151-8

[18] Mikheev LD. On the possibility of amplification of a femtosecond pulse up to the energy 1 kJ. Laser and Particle Beams. 1992;10:473-478. DOI: 10.1017/S02630346000067

[19] Aristov AI, Grudtsin YV, Zubarev IG, Ivanov NG, Konyashenko AV, Krokhin ON, Losev VF, Mavritsky AO, Mamaev SB, Mesyats GA, Mikheev LD, Panchenko YN, Rastvortseva AA, Ratakhin NA, Sentis M, Starodub AN, Tenyakov SY, Uteza O, Tcheremiskin VI, Jalovoi VI. Hybrid femtosecond laser system based on photochemical XeF(C-A) amplifier with an aperture of $12 \mathrm{~cm}$. Atmospheric and Oceanic Optics. 2009;22:1029-1034

[20] Malinovskii GY, Mamaev SB, Mikheev LD, Moskalev TJ, Sentis ML, Tcheremiskine VI, Yalovoi VI. Numerical simulation of the active medium and investigation of the pump source for the development of a photochemical $\mathrm{XeF}(\mathrm{C}-\mathrm{A})$ amplifier of femtosecond optical pulses. Quantum Electronics. 2001;31:617-622. DOI: 10.1070/ QE2001v031n07ABEH002014

[21] Clady R, Coustillier G, Gastaud M, Sentis M, Spiga P, Tcheremiskine V, Uteza O, Mikheev LD, Mislavskii V, Chambaret JP, Chẻriaux G. Architecture of a blue high contrast multiterawatt ultrashort laser. Applied Physics B-Lasers and Optics. 2006;82:347-358. DOI: 10.1007/ s00340-005-2081-1

[22] Tcheremiskine V, Uteza O, Aristov A, Sentis M, Mikheev L. Optical sources based on a multichannel surface discharge and their application to pump photolytically driven femtosecond XeF(C-A) amplifier. Proceedings of SPIE. 2008;7005:70051K. DOI: $10.1117 / 12.782779$

[23] Alexeev SV, Ivanov NG, Kovalchuk BM, Losev VF, Mesyats GA, Mikheev LD, Panchenko YN, Ratakhin NA, Yastremsky AG. Hybrid femtosecond laser system THL-100 on the base of $\mathrm{XeF}(\mathrm{C}-\mathrm{A})$ amplifier. Atmospheric and Oceanic Optics. 2012;25:221-225

[24] Losev V, Alekseev S, Ivanov N, Kovalchuk B, Mikheev L, Mesyats G, Panchenko Y, Puchikin A, Ratakhin N, Yastremsky A. Development of a 100-terawatt hybrid femtosecond laser system. Proceedings of SPIE. 2011;7393:739317. DOI: 10.1117/12.879123

[25] Losev V, Alekseev S, Ivanov N, Kovalchuk B, Mikheev L, Mesyats G, Panchenko Y, Ratakhin N, Yastremsky A. Development of a 100-terawatt hybrid femtosecond laser system on base of photochemically driven $\mathrm{XeF}(\mathrm{C}-\mathrm{A})$ amplifier. Proceedigs of . SPIE. 2012;1461:116-119. DOI: 10.1063/1.4736773

[26] Mikheev LD, Stavrovskii DB, Zuev VS. Photodissociation XeF laser operating in the visible and UV regions. Journal of Russian Laser Research. 1995;16:427-475. DOI: 10.1007/ BF02581226

[27] Eckstrom DJ, Walker HC. Multijoul performance of the photolytically pumped XeF(C-A) laser. IEEE Journal of Quantum Electronics. 1982;QE18:176-181. DOI: 10.1109/ JQE.1982.1071517 
[28] Mikheev LD, Tcheremiskine VI, Uteza OP, Sentis ML. Photochemical gas lasers and hybrid (solid/gas) blue-green femtosecond systems. Progress in Quantum Electronics. 2012;36:98-142. DOI: 10.1016/j. pquantelec.2012.03.004

[29] Alekseev SV, Aristov AI, Ivanov NG, Kovalchuk BM, Losev VF, Mesyats GA, Mikheev LD, Panchenko YN, Ratakhin NA. Multiterawatt femtosecond laser system in the visible with photochemically driven $\mathrm{XeF}(\mathrm{C}-\mathrm{A})$ boosting amplifier. Laser and Particle Beams. 2013;31:17-21. DOI: 10.1017/S0263034612000 870

[30] Alekseev SV, Aristov AI, Grudtsyn YV, Ivanov NG, Koval'chuk BM, Losev VF, Mamaev SB, Mesyats GA, Mikheev LD, Panchenko YN, Polivin AV, Stepanov SG, Ratakhin NA, Yalovoi VI, Yastremskii AG. Visible-range hybrid femtosecond systems based on a $\mathrm{XeF}(\mathrm{C}-\mathrm{A})$ amplifier: State of the art and prospects. Quantum Electronics. 2013;43:190200. DOI: 10.1070/QE2013v043n03ABEH015096

[31] Ivanov NG, Kovalenko SE, Losev VF, Panchenko YN. The laser system on XeCl molecules with high spectral brightness. Atmospheric and Oceanic Optics. 1995;8:1590-1594

[32] Byskov YI, Losev VF, Panchenko YN. Experimental investigation of the efficiency of phase conjugation of a $\mathrm{XeCl}$ laser-beam by stimulated brillouin-scattering. Kvantovaya Elektronika (Moscow). 1992;19:688-690

[33] Losev VF, Panchenko YN. Compression of a XeCl laser-pulse using stimulated brillouinscattering. Kvantovaya Elektronika (Moscow). 1994;21:55-56

[34] Baksht EH, Losev VF, Panchenko AN, Panchenko YN, Tarasenko VF. XeCl master oscillator with 300 ns pulse duration. Proceedings of SPIE. 2002;4747:88-92. DOI: 10.1117/12.460106

[35] Baksht EH, Panchenko AN, Tarasenko VF. Discharge lasers pumped by generators with inductive energy storage. IEEE Journal of Quantum Electronics. 1999;35:261-266. DOI: $10.1109 / 3.748826$

[36] Rukin SN. High-power nanosecond pulse generators based on semiconductor opening switches (review). Instruments and Experimental Techniques. 1999;42:439-467

[37] Konovalov IN, Losev VF, Liu J, Panchenko YN. Wide-aperture electric-discharge XeCl lasers. Proceeding of SPIE. 2004;5483:55-59. DOI: 10.1117/12.562962

[38] Konovalov IN, Losev VF, Panchenko YN, Ivanov NG, Sykhov MY. Electric-discharge XeCl laser emitting 10-J, 300-ns pulses. Quantum Electronics. 2005;35:237-240. DOI: 10.1070/QE2005v035n03ABEH 003428

[39] Ozaki T, Kieffer JC, Toth R, Fourmaux S, Bandule H. Experimental prospects at the Canadian advanced laser light source facility. Laser Particle Beams. 2006;24:101-106. DOI: $10.1017 / S 0263034606060150$

[40] Hofmann T, Sharp TE, Dane CB, WisoffPJ, Wilson WL, Tittel FK, Szabo G. Characterization of an ultrahigh peak power $\mathrm{XeF}(\mathrm{C}-\mathrm{A})$ excimer laser system. IEEE Journal of Quantum Electronics. 1992;28:1366-1375. DOI: 10.1109/3.135279 
